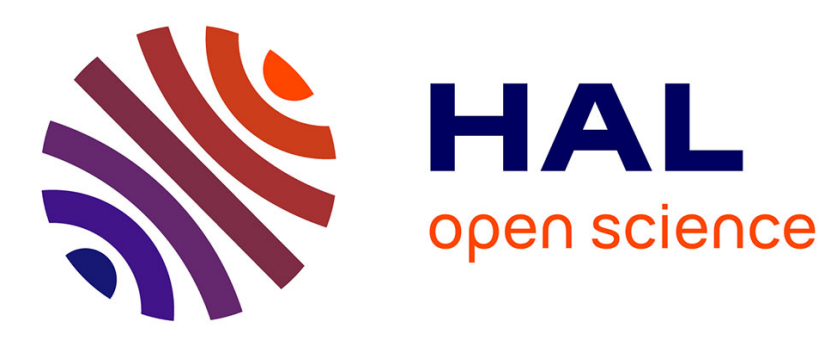

\title{
Bile acid homeostasis and intestinal dysbiosis in alcoholic hepatitis
}

Dragos Ciocan, Cosmin Sebastian Voican, Laura Wrzosek, Cindy Hugot, Dominique Rainteau, Lydie Humbert, Anne-Marie Cassard, Gabriel Perlemuter

\section{To cite this version:}

Dragos Ciocan, Cosmin Sebastian Voican, Laura Wrzosek, Cindy Hugot, Dominique Rainteau, et al.. Bile acid homeostasis and intestinal dysbiosis in alcoholic hepatitis. Alimentary Pharmacology and Therapeutics, 2018, 48 (9), pp.961-974. 10.1111/apt.14949 . hal-02022884

\section{HAL Id: hal-02022884 https: / hal.sorbonne-universite.fr/hal-02022884}

Submitted on 18 Feb 2019

HAL is a multi-disciplinary open access archive for the deposit and dissemination of scientific research documents, whether they are published or not. The documents may come from teaching and research institutions in France or abroad, or from public or private research centers.
L'archive ouverte pluridisciplinaire HAL, est destinée au dépôt et à la diffusion de documents scientifiques de niveau recherche, publiés ou non, émanant des établissements d'enseignement et de recherche français ou étrangers, des laboratoires publics ou privés. 
1 Title: Bile acid homeostasis and intestinal dysbiosis in alcoholic hepatitis

2 Running Title: Bile acid, microbiota and alcoholic hepatitis

3 Dragos Ciocan ${ }^{1}$, Cosmin Sebastian Voican ${ }^{1,2}$, Laura Wrzosek ${ }^{1,2}$, Cindy Hugot ${ }^{1}$,

4 Dominique Rainteau ${ }^{3,4,5,6}$, Lydie Humbert ${ }^{3,4,5}$, Anne-Marie Cassard ${ }^{1}$, and Gabriel

5 Perlemuter ${ }^{1,2}$

$6{ }^{1}$ Inflammation Chimiokines et Immunopathologie, DHU Hepatinov, Faculté de

7 Médecine - Université Paris-Sud/ Université Paris-Saclay, INSERM, 92140, Clamart,

8 France;

$9{ }^{2}$ AP-HP, Hepatogastroenterology and Nutrition, Hôpital Antoine-Béclère, Clamart,

10 France;

11 35orbonne Universités, UPMC Université Paris 6, Paris, France;

$12{ }^{4}$ Inflammation-Immunopathology-Biotherapy Department (DHU i2B), INSERM-ERL 13 1157, Paris, France;

14 5 UMR 7203 Laboratoire des Biomolécules, UPMC/CNRS/ENS, Paris, France;

15 'Département PM2 Plateforme de Métabolomique, APHP, Hôpital Saint Antoine, 16 Peptidomique et dosage de Médicaments, Paris, France;

17 Co-corresponding authors:

18 Prof. Gabriel Perlemuter, Service d'Hépato-Gastroentérologie et Nutrition, Hôpital 19 Antoine-Béclère, 157 rue de la Porte de Trivaux, Clamart cedex F-92141, France; 20 gabriel.perlemuter@aphp.fr.

21 Dr Anne-Marie Cassard, INSERM U996, 32 rue des Carnets, Clamart, F-92140, 22 France; cassard.doulcier@u-psud.fr

\section{Authorship Statement}

24 Gabriel Perlemuter is the submission's guarantor. Specific author contributions: DC 25 designed the project, performed the post-sequencing data processing and the 
26 statistical analysis, and wrote the manuscript. CSV designed the project and included 27 patients. LW designed the project and performed presequencing preparation 28 procedures. $\mathrm{CH}$ performed presequencing preparation procedures. DR and LH 29 performed bile acids analyses. AMC designed, performed and supervised 30 experiments and wrote the manuscript. GP designed the project, included patients, 31 supervised experiments and wrote the paper. All authors discussed the results, 32 commented and approved the final version of the manuscript.

33 Acknowledgments. The authors thank the NED group (Olivier Zemb, Béatrice 34 Gabinaud) and the GeT-PlaGe platform for their help with the sequencing data. 


\section{Summary}

36 Background: Intestinal microbiota plays an important role in bile acid homeostasis.

37 Aim: We aimed to study the structure of the intestinal microbiota and its function in 38 bile acid homeostasis in alcoholic patients based on the severity of alcoholic liver 39 disease.

40 Methods: In this prospective study, we included four groups of active alcoholic 41 patients $(\mathrm{N}=108)$ : two non-cirrhotic, with (noCir_AH, $\mathrm{n}=13$ ) or without alcoholic 42 hepatitis (noCir_noAH, n=61), and two cirrhotic, with (Cir_sAH, n=17) or without 43 severe alcoholic hepatitis (Cir_noAH, n=17). Plasma and faecal bile acids profiles, 44 and intestinal microbiota composition, were assessed.

45 Results: Plasma levels of total bile acids (84.6 vs. $6.8 \mu \mathrm{mol} / \mathrm{l}, \mathrm{p}<0.001$ ) and total 46 ursodeoxycholic acid (1.3 vs. $0.3 \mu \mathrm{mol} / \mathrm{l}, \mathrm{p}=0.03$ ) were higher in Cir_sAH than 47 Cir_noAH whereas faecal total $(2.4$ vs. $11.3, p=0.01)$ and secondary bile acids $(0.7$ 48 vs. $10.7, p<0.01)$ levels were lower. Cir_sAH patients had a different microbiota than 49 Cir_noAH patients: at the phyla level, the abundance of Actinobacteria (9 vs $1 \%$, $50 \mathrm{p}=0.01$ ) was higher and that of Bacteroidetes was lower (25 vs $40 \%, p=0.04$ ). 51 Moreover, the microbiota of Cir_sAH patients showed changes in the abundance of 52 genes involved in 15 metabolic pathways, including upregulation of glutathione 53 metabolism, and downregulation of biotin metabolism.

54 Conclusions: Patients with Cir_sAH show specific changes of the bile acid pool with 55 a shift towards more hydrophobic and toxic species that may be responsible for the 56 specific microbiota changes. Conversely, the microbiota may also alter the bile acid 57 pool by transforming primary to secondary bile acids, leading to a vicious cycle.

58 Keywords: 16S sequencing, microbiota, UDCA, biotin, glutathione, Actinobacteria, 59 Bacteroidetes 


\section{INTRODUCTION}

61 Severe alcoholic hepatitis is a life-threatening complication seen in a subset of 62 patients with alcoholic liver disease with a mortality rate of up to $25 \%$ and few 63 therapeutic options $(1,2)$. The causal role of the intestinal microbiota in the 64 development and individual susceptibility to alcoholic hepatitis has only recently been 65 shown (3-6). It has also been suggested that faecal microbiota transplantation may 66 improve gut dysbiosis and clinical outcomes in patients with cortico-resistant severe 67 alcoholic hepatitis in a recent pilot study (7). Nevertheless, the mechanisms related to 68 the role of the intestinal microbiota in alcoholic liver disease are not fully understood.

69 The relationship between bile acids and the intestinal microbiota is complex. Bile 70 acids have both direct antimicrobial effects on bacteria (8), and indirect effects 71 through their signaling proprieties which allows them to induce antimicrobial peptides 72 production (9). Detergent properties of bile acids, needed for fat digestion, influence 73 the composition of the intestinal microbiota by acting on bacterial cell membranes (8). 74 However, the diversity of the bile acids pool and enterohepatic circulation are 75 dependent on the intestinal microbiota. Indeed, primary bile acids (cholic acid, CA, 76 and chenodeoxycholic acid, CDCA) are synthesized in the liver, but secondary bile 77 acids are produced in the digestive tract. The complex pool of bile acids is then 78 reabsorbed in the portal circulation via a large panel of transporters. In addition, bile 79 acids are signaling molecules involved in regulating hepatic metabolism, 80 inflammation, and their own synthesis through the activation of various nuclear 81 receptors, such as the farnesoid X receptor (FXR) (10).

82 Chronic alcohol consumption is associated with an impaired bile acids homeostasis 83 (11-14). The level of plasma bile acids positively correlates with the histological 84 severity of $\mathrm{AH}(11)$ and is predictive of poor patient survival (15). Moreover, FXR- 
85 specific agonists attenuate chronic alcohol-induced liver injury and steatosis in 86 experimental alcoholic liver disease models $(16,17)$. Conversely, FXR-deficient mice 87 develop more severe liver injury (17). Overall, these results suggest that bile acidsdependent hepatotoxicity may be due, in part, to impaired FXR signaling.

89 We have shown in a recent work that patients with severe alcoholic hepatitis have a specific dysbiosis that renders their liver more susceptible to alcohol-induced injury

91 (4). This sensitivity was transmissible from patients to mice by intestinal microbiota 92 transplant. In these humanized mice, the bile acids pool was impaired in the feces. 93 Moreover we also showed that alcoholic patients that develop severe alcoholic 94 hepatitis have a different microbial composition as compared to patients that develop 95 other types of complications such as alcoholic pancreatitis (6).

96 However, while both the bile acids and intestinal microbiota profiles were reported in 97 alcoholic liver disease, these studies focused either only on the bile acids profile $98(11,18)$ or intestinal microbiota profile $(4)$ and never on both, in the same cohort of 99 patients or didn't included patients with severe alcoholic hepatitis $(12,13)$. In order to 100 study the relationships between intestinal microbiota modifications and bile acids 101 metabolism, we investigated herein the interplay between bile acids and intestinal 102 microbiota in well phenotyped patients at different stages of alcoholic liver disease by 103 assessing and comparing plasma and faecal bile acids profiles and intestinal 104 microbiota composition and functions in currently drinking alcoholic patients 105 according to the severity of liver lesions. 


\section{METHODS}

\section{Study subjects}

108 All patients included in this prospective study were admitted to the Hepato-

109 Gastroenterology and Nutrition Department of Antoine-Béclère University Hospital,

110 Clamart, France, for the management of excessive drinking.

111 Alcoholic patients were eligible for inclusion if they were between 17 and 75 years old

112 and had been consuming at least $50 \mathrm{~g}$ of alcohol/day and were negative for hepatitis

113 B surface antigens and hepatitis C. The exclusion criteria were gastrointestinal 114 bleeding, bacterial infection, hepatocellular carcinoma, any other carcinoma, other 115 associated severe diseases, the presence of anti-HIV antibodies, antibiotic intake in 116 the last three months, probiotic drugs use, refusal to undergo a liver biopsy if required

117 (abnormal liver function), use of any hepatoprotective treatment (UDCA, TUDCA). A 118 standardized questionnaire was used to collect information about alcohol

119 consumption (19) and patients' families were also interviewed, when possible.

120 General demographic and clinical characteristics were recorded for all patients at 121 inclusion. The study was carried out in accordance with the Helsinki Declaration and 122 was approved by the lle de France VII ethics committee (Bicêtre Hospital, 94270 le 123 Kremlin-Bicêtre, France). All patients provided written informed consent for 124 participation in the study.

125 Patients were classified into four groups:

126 - patients with alcoholic cirrhosis and severe alcoholic hepatitis (Cir_sAH, $n=$ 127 17). Severe alcoholic hepatitis was suspected in patients with a Maddrey 128 score $>32$ and was confirmed by a liver biopsy (histological score for $\mathrm{AH} \geq 6$ 129 with neutrophilic infiltration) $(4,20)$. 
- patients with alcoholic cirrhosis, but without severe alcoholic hepatitis (Cir_noAH, $n=17$ ). As the impact of non-severe alcoholic hepatitis was limited on the parameters that we studied in non-cirrhotic patients, we pooled the patients with mild alcoholic hepatitis with patients with no alcoholic hepatitis in the cirrhotic patients group.

- patients without alcoholic cirrhosis or alcoholic hepatitis (noCir_noAH, $n=61$ ),

- patients without alcoholic cirrhosis, but with alcoholic hepatitis (noCir_AH, $n=$ 13). Alcoholic hepatitis was defined by aspartate aminotransferase $>50$, aspartate aminotransferase/alanine aminotransferase $>1.5$, and both values $<$ $400 \mathrm{IU} / \mathrm{L}(20,21)$ or, if a liver biopsy was available (12/13 patients), an AH score between 3 and 5 (with neutrophilic infiltration) or $\geq 6$ (with neutrophilic infiltration and a Maddrey score <32).

Diagnosis of cirrhosis was made based on clinical examination, laboratory test, imaging and endoscopy studies or by a liver biopsy, when available. As patients with cirrhosis have a different intestinal microbiota profile than those without, and most patients with severe alcoholic hepatitis exhibit histological evidence of micronodular cirrhosis, we did not perform a global comparison between the four groups but separately compared the patients with and without cirrhosis.

\section{Biochemical assays}

Bile acids measurements in plasma of 55 patients and feces of 73 patients were performed using high-performance liquid chromatography-tandem mass spectrometry as previously described (22). Serum fibroblast growth factor-19 (FGF19) was measured for 55 patients using a sandwich ELISA kit (R\&D Systems) according to the manufacturer's instructions.

\section{Analysis of the intestinal microbiota by $16 S$ ribosomal RNA sequencing}


155 Faecal samples were available for 96 patients. The composition of the faecal

156 microbiota was analyzed by high-throughput sequencing with Illumina MiSeq

157 technology, targeting the $16 \mathrm{~S}$ ribosomal DNA V3-V4 region in the paired-end mode

158 ( 2 x 300 base pairs) (GenoToul, Toulouse), as previously described (23). Data were

159 processed with the quantitative insights into microbial ecology (QIIME v1.9.0)

160 pipeline, using its default parameters. Closed reference operation taxonomic

161 mapping was performed using the Greengenes database (v13.8, 97\% sequence

162 similarity).

163 The mean number of quality-controlled reads was $26535 \pm 7840$ (mean \pm SD) per 164 sample (minimum count: 9833, maximum count: 56968). After rarefaction at 9,000

165 reads per sample, bacterial alpha diversity was estimated on the basis of the

166 Shannon's index. OTUs with a prevalence $<5 \%$ were removed from the analysis.

167 Functional composition of the intestinal metagenome was predicted using

168 Phylogenetic Investigation of Communities by Reconstruction of Unobserved States

169 (PICRUSt) (24). This is a computational approach that accurately predicts the

170 abundance of gene families in the microbiota and thus provides information about the

171 functional composition of the microbial community. Linear discriminant analysis (LDA)

172 effect size (LEfSe) analysis was performed to identify the taxa and functions

173 displaying the largest differences in abundance in the microbiota between groups

174 (25). Only taxa and functions with an LDA score $>2$ and a significance of $<0.05$, as

175 determined by Wilcoxon signed-rank tests, are shown. LEfSe and PICRUSt were

176 accessed online (http://huttenhower.sph.harvard.edu/galaxy/).

\section{Statistical analysis}

178 The results are expressed as the means \pm SD for normally distributed data or median

179 [min, max] for non-normally distributed data. Data normality was tested for each 
180 parameter using the Shapiro-Wilk test $(p>0.05)$. Unpaired t-tests or Mann-Whitney

181 U-tests were used to compare continuous data between groups, depending on the

182 data distribution. Chi2 or Fisher's exact tests were used to compare discrete

183 parameters between groups. The Spearman correlation test was used to find

184 correlations between bile acids and intestinal microbiota. Benjamini-Hochberg false

185 discovery rate (FDR) correction was used to correct for multiple hypothesis testing,

186 when applicable. A p-value $<0.05$ was considered to be statistically significant. The

187 comparisons were performed with R software v2.14.1 unless stated otherwise.

188 Bile acids data were processed and analyzed in MetaboAnalyst

189 (http://www.metaboanalyst.ca) (26) using supervised and unsupervised methods:

190 Principal Component Analysis (PCA) and Partial Least Squares Discriminant

191 Analysis (PLS-DA). Data were log transformed and pareto-scaled and the results

192 validated using leave-one-out cross-validation procedures. 


\section{RESULTS}

\section{Demographic and laboratory data}

195 A total of 108 patients were included in the study. We classified patients into four 196 groups: patients with alcoholic cirrhosis and severe alcoholic hepatitis $(\mathrm{Cir}$ s $\mathrm{AH}, \mathrm{n}=$

197 17); patients with alcoholic cirrhosis, but without severe alcoholic hepatitis (Cir_noAH, $198 \mathrm{n}=17$ ); patients without alcoholic cirrhosis or alcoholic hepatitis (noCir_noAH, $\mathrm{n}=$ 199 61); and patients without alcoholic cirrhosis, but with alcoholic hepatitis (noCir_AH, n $200=13)$.

201 The demographic and laboratory data are summarized in Table 1. There was no 202 difference in age, sex, body mass index (BMI), or duration of alcohol intake between 203 the groups. As expected, patients with noCir_AH had higher aspartate 204 aminotransferase (AST), alanine aminotransferase (ALT), total bilirubin, gamma205 glutamyl transferase (GGT), and C-reactive protein levels than noCir_noAH patients. 206 Cir_sAH patients had lower alcohol consumption than the Cir_noAH patients and 207 higher total bilirubin and C-reactive protein levels, a higher MELD score, and lower 208 albumin levels.

209 Intestinal microbiota profiles

210 We first studied interindividual bacterial diversity (beta diversity). Cir_sAH patients 211 had a different intestinal microbiota structure (proportion of bacteria) than Cir_noAH 212 patients (weighted UNIFRAC distances, $R=0.09, p=0.04$ ) (Figure 1A). There was 213 no difference in the overall bacterial composition of the intestinal microbiota between 214 these two groups (unweighted UNIFRAC distances, $R=0.05, p=0.1$ ) (Figure 1B). 215 This result suggests that the two groups have an intestinal microbiota with similar 216 bacterial species, but with different relative abundances. There was no difference in 217 the beta diversity between noCir_noAH and noCir_AH patients (data not shown). 
218 There was also no difference in the intra-individual bacterial diversity (alpha diversity) 219 either between the noCir_noAH and noCir_AH groups, nor the Cir_sAH and 220 Cir_noAH groups, measured by various indices (observed OTUs, Shannon index, 221 Chao index, and PD whole tree index, data not shown).

222 At the phyla level, Cir_sAH patients had a higher abundance of Actinobacteria and 223 lower abundance of Bacteroidetes than Cir_noAH patients. Among Actinobacteria, 224 Cir_sAH patients had a higher abundance of Actinomyces, Rothia, and 225 Bifidobacterium than Cir_noAH patients. Among Proteobacteria, Cir_sAH patients 226 had a higher abundance of Haemophilus and Enterobacteriaceae and a lower 227 abundance of Bilophila than Cir_noAH patients. Cir_sAH patients also had a lower 228 relative abundance of Parabacteroides (Bacteroidetes phylum), Oscillospira, and 229 Christensenellaceae families (Firmicutes phylum) and a higher relative abundance of 230 Lactobacillus and Lactococcus (Firmicutes phylum) than Cir_noAH patients (Figure 231 1C and Supplemental Table 1).

232 Although there was no difference in the overall composition of the intestinal 233 microbiota between noCir_AH and noCir_noAH patients, LEFsE analysis showed 234 that noCir_AH patients had a higher abundance of Dorea (Firmicutes phylum), 235 Wolbachia (Proteobacteria phylum) and Rothia (Actinobacteria phylum) than 236 noCir_noAH patients (Figure 1D). These results suggest that a specific dysbiosis is 237 associated with hepatic inflammation in $\mathrm{AH}$ in both patients with and without cirrhosis 238 and independently of alcohol consumption.

239 Functional Intestinal Metagenome Prediction in Alcoholic Hepatitis:

240 The dysbiosis identified in Cir_sAH patients prompted us to also examine the 241 metabolic pathways associated with this specific intestinal microbiota. The intestinal 
242 microbiota of the Cir_sAH group had a higher proportion of metabolic pathways 243 containing gene functions, such as glutathione metabolism, membrane transport 244 (phosphotransferase system), and nucleotide metabolism than that of the Cir_noAH 245 group. The intestinal microbiota of Cir_sAH patients also had a lower proportion of 246 genes for energy metabolism (methane metabolism and carbon fixation pathways in 247 prokaryotes), amino acid metabolism (arginine, proline and histidine metabolism), 248 lipid metabolism (lipid biosynthesis proteins), glycan biosynthesis and metabolism, 249 metabolism of cofactors and vitamins (biotin metabolism), metabolism of terpenoïds 250 and polyketides (polyketide sugar unit biosynthesis), biosynthesis of other secondary 251 metabolites (streptomycin biosynthesis), and of the transcription machinery than that 252 of Cir_noAH patients (Figure 1E). There was no difference between the two groups in 253 the secondary bile acids biosynthesis pathway.

254 These results indicate that the dysbiosis observed in patients with severe alcoholic 255 hepatitis is also associated with a shift in the bacterial metabolic pathways.

\section{Bile acids}

257 Bile acids can shape the intestinal microbiota, and in turn, the intestinal microbiota 258 alters the bile acids pool. Thus, we studied plasma bile acids and faecal bile acids 259 profiles and their relationship with the dysbiosis observed in alcoholic patients with 260 and without alcoholic cirrhosis.

\section{$261 \quad$ Plasma bile acid profile}

262 We first studied the plasma bile acids profile associated with liver inflammation in 263 alcoholic patients. Cir_sAH patients had a different plasma bile acids profile than 264 Cir_noAH patients, as shown by PCA (Figure 2A) and on a heatmap (Supplementary 265 figure 1A). Cir_sAH patients had higher levels of total bile acids, total primary bile 
266 acids, total conjugated bile acids (total glyco- and tauroconjugated bile acids), 267 primary glyco- and tauroconjugated bile acids, total CA (glycocholate (GCA) and 268 taurocholate (TCA)), total CDCA (glycochenodeoxycholate (GCDCA) and 269 taurochenodeoxycholate (TCDCA)), and total UDCA (tauroursodeoxycholate 270 (TUDCA)) than Cir_noAH patients (Figures 2 B-D and Supplementary Table 2). 271 Because of the higher total bile acids levels in Cir_sAH patients, we also studied the 272 relative proportion of each bile acids (bile acids concentration/total bile acids 273 concentration) between the two groups. Cir_sAH patients had a higher relative 274 proportion of total primary bile acids, total CDCA (TCDCA), total primary conjugated 275 bile acids, primary tauroconjugated bile acids, and TUDCA than Cir_noAH patients. 276 They also had a lower relative proportion of total sulphoconjugated bile acids, total 277 secondary bile acids, total secondary conjugated bile acids, secondary 278 glycoconjugated bile acids, total lithocholate (LCA) (LCA, glycolithocholate (GLCA), 279 lithocholate-3-sulfate (LCA3s), taurolithocholate-3-sulfate (TLCA3s), and 280 glycolithocholate-3-sulfate (GLCA3s)), total deoxycholate (DCA) (DCA; 281 glycodeoxycholate (GDCA), and taurodeoxycholate (TDCA)), chenodeoxycholic acid 282 3-sulfate (CDCA3s), and glycoursodeoxycholate-3-sulfate (GUDCA3s) than 283 Cir_noAH patients (Figures 2 B, C, E).

284 We then used PLS-DA to find the plasma bile acids that best discriminate between 285 the two groups. The model showed a significant distinction $(\mathrm{R} 2=0.6, \mathrm{Q} 2=0.4$, 286 prediction accuracy during training: $p=0.001,1000$ permutations) between the 287 Cir_sAH and Cir_noAH groups (Figure 3A). The average AUROC confirmed that the 288 model was able to discriminate Cir_noAH from Cir_sAH patients $(0.955,95 \% \mathrm{Cl}$ : 289 0.763-1, Supplementary Figure 2A). TUDCA was the most discriminant bile acids 
290 between the two groups according to the PLS-DA (variable importance in projection, 291 VIP $=2.1$, Figure 3B).

292 Comparison of the bile acids profile of noncirrhotic patients showed higher levels of 293 TUDCA in noCir_AH patients than noCir_noAH patients. Moreover, noCir_AH 294 patients had higher proportions of TUDCA and total conjugated bile acids and a 295 lower proportion of DCA than noCir_AH patients (data not show). However, these 296 changes were no longer significant after correction for multiple comparisons.

297 These results indicate an increase in the pool of bile acids in Cir_sAH patients 298 Moreover, the increase in TUDCA levels, a bile acids produced exclusively by the 299 intestinal microbiota from CDCA, and that of primary bile acids s are consistent with a 300 shift in bile acids transformation in the gut.

\section{$301 \quad$ Faecal bile acids}

302 We further studied the bile acids profile in Cir_sAH patients. Cir_sAH patients had a 303 different faecal bile acids profile than Cir_noAH patients (Figure 4A and 304 Supplementary Figure 1B). Cir_sAH patients had lower total faecal bile acids, total 305 unconjugated bile acids, total glycoconjugated bile acids, total secondary bile acids, 306 secondary unconjugated bile acids, secondary glyco- and tauroconjugated bile acids, 307 total LCA (LCA), and total DCA (DCA, GDCA, TDCA, deoxycholate 3-sulfate: 308 DCA3s) than Cir_noAH patients (Figures 4B-D and Supplementary Table 3). We also 309 examined the relative amount of each bile acids (faecal bile acids/total faecal bile 310 acids). Cir_sAH patients had a higher percentage of total primary bile acids, total 311 CDCA (CDCA, TCDCA), primary unconjugated bile acids, and CA than Cir_noAH 312 patients and a lower percentage of total secondary bile acids, total secondary 
313 unconjugated bile acids, secondary glycoconjugated bile acids, secondary

314 tauroconjugated bile acids, and total DCA (DCA, GDCA, TDCA) (Figures 4 B, C, E).

315 PLS-DA showed a significant distinction $(\mathrm{R} 2=0.8, \mathrm{Q} 2=0.6$, prediction accuracy 316 during training: $p=0.001,1000$ permutations) between the Cir_sAH and Cir_noAH 317 groups (Figure 5A). The average AUROC confirmed that the model was able to 318 discriminate Cir_noAH from Cir_sAH patients $(0.977,95 \%$ Cl: $0.769-1)$ 319 (Supplementary Figure 2B). Secondary glycoconjugated bile acids and GDCA were 320 the most discriminant bile acids between the two groups according to the PLS-DA 321 (variable importance in projection, VIP = 2.1 and 2.1, respectively, Figure 5B).

322 Comparison of the faecal bile acids profiles between noncirrhotic patients showed the 323 observed changes (higher taurolithocholate, TLCA, and GLCA ratios in noCir_AH 324 than noCir_noAH patients) to no longer be significant after correction for multiple 325 comparisons (data not show), as observed in plasma.

326 These results confirm the decrease in bile acids excretion and impaired bile acids 327 transformation in the gut by the intestinal microbiota of Cir_sAH patients relative to 328 that of Cir_noAH patients.

329 The relationship between intestinal microbiota and bile acids homeostasis

330 We assessed the correlation between the bile acids profiles with the bacteria species 331 identified in the feces, as the composition and quantity of the bile acids pool influence 332 the intestinal microbiota, and conversely, the metabolism of bile acids is dependent 333 on intestinal microbiota composition. Primary and secondary plasma bile acids levels 334 positively correlated with most of the taxa in Cir_noAH patients (Figure 6A) while 335 primary plasma bile acids negatively correlated with taxa from Bacteroidetes and 336 Firmicutes phyla in Cir_sAH patients (Figure 6B). Total UDCA, TUDCA, and 
337 glycoursodeoxycholate (GUDCA) positively correlated with most of the taxa in

338 Cir_noAH patients while it was negatively correlated with taxa from the Cir_sAH 339 patients (Figure 6A and 6B).

340 Faecal primary bile acids were mostly negatively correlated with most of the taxa in

341 Cir_noAH patients (Figure 6C) while in Cir_sAH patients, primary faecal bile acids

342 were negatively correlated and secondary faecal bile acids were positively correlated

343 with most of the taxa (Figure 6D)

344 These correlations suggest that the abundance of bacteria carrying the enzymes

345 needed for bile acids deconjugation and transformation into secondary bile acids and

346 UDCA is reduced in the intestinal microbiota of Cir_sAH patients.

\section{FXR-FGF-19 in severe alcoholic hepatitis}

348 As signaling molecules, bile acids activate ileal FXR and induce the production of

349 FGF19. Cir_sAH patients had higher plasma levels of FGF19 than Cir_noAH patients 350 (282 \pm 431 vs. $55 \pm 75 \mathrm{pg} / \mathrm{mL}, \mathrm{p}=0.03$, Table 1). In patients without cirrhosis, 351 plasma levels of FGF19 were higher in patients with $\mathrm{AH}$ patients than those without,

352 but did not reach statistical significance (154 \pm 368 vs. $69 \pm 74 \mathrm{pg} / \mathrm{mL}, \mathrm{p}=0.5$ ).

353 However, FGF-19 positively correlated with the MELD score $(r=0.49, p=0.04)$, but 354 not the Maddrey or AH histological scores.

355 These results suggest that FXR is activated in Cir_sAH patients independently of the 356 faecal bile acids concentration in the gut. 


\section{DISCUSSION}

358 In this study, we characterized the intestinal microbiota, its functions, and its

359 relationship with bile acids homeostasis in well phenotyped alcoholic liver disease

360 patients, in order to overcome potential confounders such as alcoholic liver disease

361 stage and previous or concomitant treatments. Moreover, as alcohol induces a

362 specific dysbiosis in both animal models of alcoholic liver disease $(4,23,27)$ and

363 humans $(12,13,28)$, including higher levels of some members of Proteobacteria and

364 lower Bacteroidaceae, Lachnospiraceae, and Prevotellaceae, we only compared

365 patients with ongoing alcohol consumption. This allowed us to identify specific

366 changes related only to the liver disease, independently on the amount and duration

367 of alcohol consumption.

368 Alcoholic hepatitis did not modify the overall composition of the intestinal microbiota

369 in patients without cirrhosis. A similar result has been recently reported in a mouse

370 model of acute-on-chronic alcohol feeding (29). However, we observed an increase

371 in the abundance of Dorea, Wolbachia and Rothia in noCir_AH patients. Among

372 patients with alcoholic cirrhosis, severe alcoholic hepatitis patients had higher

373 abundance of bacteria of the Actinobacteria phylum including the Actinomyces,

374 Rothia, and Bifidobacterium genus. The abundance of Lactobacillus (Firmicutes

375 phylum), Haemophylus (from the Pasteurellaceae family, Proteobacteria phylum),

376 and an unidentified member of the Enterobacteriaceae family (Proteobacteria

377 phylum) was also higher. Conversely, the abundance of bacteria of the Bacteroidetes

378 phylum was lower. Interestingly, these changes are consistent with data from other

379 studies that investigated the intestinal microbiota in alcoholic liver disease and in

380 other liver diseases such as NAFLD (28-32), suggesting that these changes may be

381 related to cirrhosis and impaired liver function rather than to the cause of liver 
382 disease. These results also confirms the increase seen in Bifidobacterium genus in

383 severe alcoholic hepatitis patients that we previousely reported in a smaller sample of

384 severe alcoholic hepatitis patients (4). Furthermore, by increasing the number of 385 patients included in the present study we also identifies new taxa associated with 386 severe alcoholic hepatitis as compared to our previous study.

387 We further explored the role of the intestinal microbiota in Cir_sAH using PICRUSt to 388 predict the metagenomic profile of the intestinal microbiota. We observed a switch in 389 the functions of the intestinal microbiota in Cir_sAH patients, including a decrease in 390 the biotin metabolic pathway. Biotin is a member of the vitamin-B family of vitamins 391 and acts as a cofactor for several carboxylases in mitochondria. Exogenous biotin is 392 obtained from dietary sources or intestinal biotin-producing bacteria (33). Plasma 393 biotin levels in chronic alcohol patients are reduced due to inhibition of carrier394 mediated biotin transport in the jejunum and colon (34). Thus, reduced production in 395 the gut and decreased absorption in alcoholic patients could lead to the dysfunction 396 of mitochondria, which could impair the hepatic response to inflammation in severe 397 alcoholic hepatitis.

398 We also observed altered glutathione metabolism in the intestinal microbiota of 399 Cir_sAH patients. Glutathione is a powerful antioxidant and patients with alcoholic 400 liver disease have low hepatic and plasma glutathione levels (35). In this context, it 401 has been shown that the bile salt hydrolase $(\mathrm{BSH})$ gene from Bifidobacterium longum 402 is co-transcribed with the gene encoding glutamine synthetase adenylyltransferase 403 ( $g \operatorname{lnE})$, a component of the nitrogen regulation cascade (36). Thus, the increase in 404 the abundance of Bifidobacterium in Cir_sAH patients could be responsible, at least 405 partially, for the increased proportion of primary unconjugated faecal bile acids, as a 
406 result of $\mathrm{BSH}$ activity and increased glutathione metabolism of the intestinal 407 microbiota of these patients.

408 It has been suggested, in a previous work, that an increase in primary bile acids was 409 associated with the severity of histological lesions in $\mathrm{AH}$ (11). A similar result was 410 observed in our study. Of note, in the previous work (11), the authors provided an 411 overall plasma bile acids profile in patients with $\mathrm{AH}$ ranging from mild $\mathrm{AH}$ to severe 412 alcoholic hepatitis and irrespective of the presence of cirrhosis, that was present in $41375 \%$ of their patients. As alcoholic cirrhosis is associated with an impaired bile acids 414 profile $(12,13)$, their results might be biased by the mix of cirrhotic and non-cirrhotic 415 patients. Moreover, according to the method used for bile acids assay (HPLC), they 416 could only detect primary and secondary bile acids but neither their conjugated forms 417 nor UDCA. Other studies have investigated plasma bile acids, faecal bile acids and 418 intestinal microbiota in alcoholic patients $(12,13)$. They suggested, that alcohol intake 419 in both cirrhosis and non-cirrhotic patients is associated with a decrease in 420 conjugated CDCA in plasma (12). In our study, CDCA was increased in severe 421 alcoholic hepatitis, suggesting that this increase is due to liver inflammation (ie 422 severe alcoholic hepatitis) independently of the presence of cirrhosis of alcohol 423 intake. We also suggest that perturbation of intestinal microbiota is involved in the 424 specific modifications bile acids metabolism observed in patients with severe 425 alcoholic hepatitis.

426 More hydrophobic bile acids (CA, CDCA, DCA) rapidly induce apoptosis (37), 427 whereas less hydrophobic bile acids (UDCA) are less toxic (38). Moreover, total 428 plasma bile acids and primary plasma bile acids (CA and CDCA) levels have been 429 shown to positively correlated with the $\mathrm{AH}$ severity and steatosis $(11,18,39)$. Several 430 mechanisms may explain the increase of the bile acids pool, including upregulation of 
431 cholesterol 7a-hydroxylase (Cyp7A1) induced by both chronic and acute alcohol 432 consumption (40) and/or a decrease in bile acids excretion in the bile and 433 subsequent release in the plasma in the context of $\mathrm{AH}$. Moreover, it has also been 434 suggested that the intestinal microbiota can contribute to biliary inflammation (41), 435 which could impair bile acids circulation. These hypotheses are supported by the 436 increased levels of primary and conjugated plasma bile acids in the Cir_sAH patients 437 and decrease in the proportion of secondary plasma bile acids and total faecal bile 438 acids. We can thus hypothesize that the excess plasma bile acids do not reach the 439 gut where they could be deconjugated and transformed into secondary bile acids. 440 Moreover, conjugation of either taurine or glycine to bile acids decreases their 441 hydrophobicity and thus their toxicity. There was also a trend towards switching the 442 plasma bile acids pool from gylcoconjugated forms towards tauroconjugated forms, 443 which are less toxic.

444 CDCA is the most potent FXR agonist capable of inducing FGF19 expression (42). 445 Here, plasma FGF-19 levels were higher in Cir_sAH than Cir_noAH patients. FGF-19 446 is produced in the ileum by FXR activation. Faecal bile acids activates FXR and acts 447 in a negative feedback loop by blocking CYP7A1 and bile acid synthesis (classical 448 pathway). FGF-19 is absent from primary, non-activated hepatocytes, but bile acids 449 activated hepatic FXR can induce FGF19 secretion in vitro (43) and in vivo in patients 450 with cholestasis (44) by an autocrine/paracrine mechanism, independently of SHP $451(43,44)$. However, a recent study found that FGF19 levels were significantly elevated 452 in patients with alcoholic hepatitis while serum 7-alpha-hydroxy-4-cholesten-3-one 453 (C4) levels, a bile acids synthesis marker for de novo synthesis was decreased 454 suggesting a prominent role of cholestasis (14). Moreover, the authors showed that in 455 alcoholic hepatitis FGF-19 originates in cholangiocytes and ductular cells from 
456 smaller ductules (progenitor cells). Here, FGF-19 positively correlated with the MELD 457 score. This is consistent with other studies that reported a correlation between FGF45819 levels and liver disease severity $(14,44)$ and suggests that the increase in FGF-19 459 levels observed in our study has a double origin, hepatic (due to increased plasma 460 bile acids, dominated by CDCA) and intestinal. Indeed, there was a shift of the faecal 461 bile acids pool in the gut toward species with a higher affinity for FXR, as shown by 462 the increase in the proportion of CDCA, although there was an overall decrease in 463 total faecal bile acids. Thus, high levels of FGF-19 could increase CDCA synthesis by 464 directing bile acids synthesis from the classic (neutral) to the alternative (acidic) 465 pathway, due to the blockade of CYP7A1, but not of cholesterol 7 $\beta$-hydroxylase 466 (CYP7B1). Therefore, high levels of FGF-19 are probably insufficient to counteract 467 the increased bile acids synthesis induced by alcohol in alcoholic liver disease. 468 Moreover, it promotes the shift of the bile acids pool towards more hydrophobic, toxic 469 species.

470 There was also an increase in total plasma UDCA and TUDCA levels in Cir_sAH 471 patients relative to $\mathrm{Cir}$ _noAH patients. These bile acids have hepatoprotective 472 effects. However, TUDCA exerts this effect by replenishing hepatic mitochondrial 473 glutathione (45). Thus, the increase in glutathione metabolism of the intestinal 474 microbiota, which may decrease its bioavailability to the mitochondria, combined with 475 an intestinal microbiota-associated decrease in the levels of biotin, an essential 476 cofactor of mitochondrial metabolism, could explain why the increased TUDCA levels 477 seen in Cir_sAH patients does not have a hepatoprotective effect. This is supported 478 by the fact that UDCA showed hepatoprotective effects in in vitro studies and early 479 stages of alcoholic liver disease, but not in severe alcoholic hepatitis patients with 480 cholestasis (46). 
481 Specific bacteria may be involved in the production of UDCA in severe alcoholic 482 hepatitis patients. Plasma UDCA, that was increased in severe alcoholic hepatitis 483 patients, and that is only produced by bacteria in the gut from CDCA, positively 484 correlated with the abundance of Actinobacteria and Proteobacteria phyla. The 485 abundance of these phyla was increased in severe alcoholic hepatitis patients, as 486 was the abundance of the Bifidobacteria and Clostridium genera. Administration of 487 Bifidobacteria animalis, as a bile salt-hydrolysing bacteria, and Clostridium absonum, 488 as a CDCA to UDCA epimerizing bacteria, result in increased levels of faecal UDCA 489 in pigs (47).

490 An increased level of faecal bile acids was reported in cirrhotic patients with ongoing 491 alcohol consumption but, in these patients, the consequences of a potential liver 492 inflammatory process (ie alcoholic hepatitis) is unknown $(12,13)$. We now show that 493 total faecal bile acids levels probably decreased in Cir_sAH patients due to 494 decreased excretion of plasma bile acids in the bile, as discussed earlier. Faecal bile 495 acids shape the intestinal microbiota as deconjugation provides cellular carbon, 496 nitrogen, and sulfur for some bacterial species, especially Bacteroides and Bilophila 497 (48). Thus, the decrease in faecal bile acids levels in Cir_sAH patients may be 498 responsible for the decrease in the abundance of Bacteroidetes and Bilophila. 499 Moreover, primary faecal bile acids levels have been shown to increase intestinal 500 permeability (49), which can increase PAMP release into the systemic circulation, 501 participating in the higher levels of endotoxemia observed in alcoholic liver disease 502 patients. Furthermore, bile acids bactericidal activity is related to their hydrophobicity, 503 which increases their affinity for the phospholipid bilayer of the bacterial cell 504 membrane, and unconjugated bile acids are week acids with strong bactericidal 505 activities. Among the bile acids, DCA is extremely toxic and inhibits the growth of 
506 many intestinal bacteria, including Clostridium perfringens, Bacteroides fragilis, 507 Lactobacilli, and Bifidobacteria $(50,51)$. In our study we observed a decrease in 508 unconjugated bile acids, total secondary bile acids and DCA in the feces, that are 509 highly hydrophobic. This may be responsible for the increase levels of Lactobacillus 510 and Bifidobacterium seen in the microbiota of these patients. It has also been 511 suggested that a decreased level of faecal bile acids stimulated the growth of gram512 negative and conversely decreases the growth gram-positive bacteria (52). Indeed, 513 we observed in severe alcoholic hepatitis patients an increase in several taxa that are 514 gram-negative (eg Gammaproteobacteria) that could be secondary to the decrease 515 faecal bile acids level. Moreover, gram-negative bacteria produce LPS that was 516 related to increases alcoholic liver necrosis and inflammation (53). We also observed 517 in our study a decrease in several taxa from the gram-positive Firmicutes phylum 518 (Christensenellaceae, Oscillospira) that 7a-dehydroxylate primary bile acids to toxic 519 secondary bile acids. Thus, the decrease and shift of the bile acids pool in the feces 520 could be responsible for the increase in LPS-producing bacteria and for the decrease 521 of gram-positive members of Firmicutes able to transform primary bile acids into 522 secondary bile acids. This hypothesis may explain the decrease in secondary bile 523 acids in severe alcoholic hepatitis patients observed in our study.

524 A limit of our study was a potential lack of power related to the small number of 525 patients, which did not allow us to identify changes in taxa with low counts. However, 526 the recruitment of severe alcoholic hepatitis patients for intestinal microbiota studies 527 is challenging, as most are rapidly treated (often by antibiotics to prevent or treat 528 complications). This bias did not occur in our patients as they were included before 529 any specific treatment for severe alcoholic hepatitis. Moreover, we did not exclude 530 patients with proton-pump inhibitors intake which was shown to alter the IM 
531 composition (54). However, there was no difference in the use of proton-pump 532 inhibitors between groups, suggesting that they are not responsible for the results 533 observed in our study.

534 In conclusion, severe alcoholic hepatitis is associated with specific alterations of the 535 bile acids homeostasis and of the intestinal microbiota. These changes are 536 characterized by an increased level of hydrophobic bile acids and of Actinobacteria 537 and a decrease of Bacteroidetes. The increase and shift in the bile acids pool 538 towards hydrophobic and toxic species could be responsible for the specific intestinal 539 microbiota changes, including an increase in the LPS-producing gram-negative 540 bacteria such as Gammaproteobacteria and a decrease in certain gram-positive 541 bacteria capable to transform primary into secondary bile acids. Furthermore, the 542 changes in the intestinal microbiota were associated with a shift in its functions, 543 especially decreased biotin metabolism and increased glutathione metabolism, which 544 could play a role in the initiation and progression of severe alcoholic hepatitis, 545 through impairment of the protective effects of UDCA on mitochondrial metabolism. 546 Our study provides a new hypothesis for future studies to address bile acids and the 547 intestinal microbiota as new therapeutic targets to improve the management of 548 alcoholic liver disease patients.

\section{Grant Support:}

550 This work was supported by INSERM, Univ Paris-Sud, SNFGE (Société 551 Nationale Francophone de Gastro-entérologie), AFEF (French Association for the 552 Study of Liver), IREB/FRA (Institut de Recherches sur les Boissons/Fondation pour la 553 recherche en alcoologie), IRIS (Institut de Recherches Internationales Servier), 554 ERAB (The European Foundation for Alcohol Research), FRM (Fondation pour la 
555 Recherche Médicale) and Biocodex. INSERM U996 is a member of the Laboratory of 556 Excellence LERMIT supported by a grant from the Agence Nationale de la 557 Recherche (ANR-10-LABX-33). DC was supported by research fellowship from 558 INSERM

559 Disclosures:

560 DC has received travel funds from Biocodex.

561 GP has, in the last three years, received travel funds from Abbvie, Gilead, Biocodex 562 and Adare, consulting fees from Biocodex, Gilead, Servier, Adare and Pileje, and 563 royalties from Elsevier-Masson, John Libbey Eurotext, Solar and 564 Flammarion/Versilio.

565 AMC has received travel funds from Biocodex and royalties from Elsevier-Masson, 566 John Libbey Eurotext and Flammarion/Versilio. 


\section{References}

569 1. Orrego H, Blake JE, Blendis LM, Medline A. Prognosis of alcoholic cirrhosis in the

570 presence and absence of alcoholic hepatitis. Gastroenterology. janv 1987;92(1):208-14.

571 2. European Association for the Study of Liver. EASL clinical practical guidelines:

572 management of alcoholic liver disease. J Hepatol. août 2012;57(2):399-420.

573

574

575

576

577

578

3. Yan AW, Fouts DE, Brandl J, Stärkel P, Torralba M, Schott E, et al. Enteric dysbiosis associated with a mouse model of alcoholic liver disease. Hepatology. janv 2011;53(1):96-105.

4. Llopis M, Cassard AM, Wrzosek L, Boschat L, Bruneau A, Ferrere G, et al. Intestinal microbiota contributes to individual susceptibility to alcoholic liver disease. Gut. mai 2016;65(5):830-9.

5. Cassard A-M, Ciocan D. Microbiota, a key player in alcoholic liver disease. Clin Mol Hepatol. 22 déc 2017;

6. Ciocan D, Rebours V, Voican CS, Wrzosek L, Puchois V, Cassard A-M, et al. Characterization of intestinal microbiota in alcoholic patients with and without alcoholic hepatitis or chronic alcoholic pancreatitis. Sci Rep. 19 mars 2018;8(1):4822.

7. Philips CA, Pande A, Shasthry SM, Jamwal KD, Khillan V, Chandel SS, et al. Healthy Donor Fecal Microbiota Transplantation in Steroid-Ineligible Severe Alcoholic Hepatitis: A Pilot Study. Clin Gastroenterol Hepatol. avr 2017;15(4):600-2.

587

588

589

590

592

593

594

595

596

597

598

599

600

601

602

8. Begley M, Gahan CGM, Hill C. The interaction between bacteria and bile. FEMS Microbiol Rev. sept 2005;29(4):625-51.

9. Inagaki T, Moschetta A, Lee Y-K, Peng L, Zhao G, Downes M, et al. Regulation of antibacterial defense in the small intestine by the nuclear bile acid receptor. Proc Natl Acad Sci USA. 7 mars 2006;103(10):3920-5.

10. Fickert $\mathrm{P}$, Wagner M. Biliary bile acids in hepatobiliary injury - What is the link? J Hepatol. sept 2017;67(3):619-31.

11. Trinchet JC, Gerhardt MF, Balkau B, Munz C, Poupon RE. Serum bile acids and cholestasis in alcoholic hepatitis. Relationship with usual liver tests and histological features. J Hepatol. août 1994;21(2):235-40.

12. Kakiyama G, Hylemon PB, Zhou H, Pandak WM, Heuman DM, Kang DJ, et al. Colonic inflammation and secondary bile acids in alcoholic cirrhosis. Am J Physiol Gastrointest Liver Physiol. 1 juin 2014;306(11):G929-937.

13. Bajaj JS, Kakiyama G, Zhao D, Takei H, Fagan A, Hylemon P, et al. Continued Alcohol Misuse in Human Cirrhosis is Associated with an Impaired Gut-Liver Axis. Alcohol Clin Exp Res. nov 2017;41(11):1857-65. 
603

604

605

606

607

608

609

610

611

612

613

614

615

616

617

618

619

620

621

622

623

624

625

626

627

628

629

630

631

632

633

634

635

636

637

638

639

640
14. Brandl K, Hartmann P, Jih LJ, Pizzo DP, Argemi J, Ventura-Cots M, et al. Dysregulation of serum bile acids and FGF19 in alcoholic hepatitis. J Hepatol. 12 avr 2018;

15. Nissenbaum M, Chedid A, Mendenhall C, Gartside P. Prognostic significance of cholestatic alcoholic hepatitis. VA Cooperative Study Group \#119. Dig Dis Sci. juill 1990;35(7):891-6.

16. Lívero F a. R, Stolf AM, Dreifuss AA, Bastos-Pereira AL, Chicorski R, Oliveira LG, et al. The FXR agonist 6ECDCA reduces hepatic steatosis and oxidative stress induced by ethanol and low-protein diet in mice. Chem Biol Interact. 25 juin 2014;217:19-27.

17. Wu W, Zhu B, Peng X, Zhou M, Jia D, Gu J. Activation of farnesoid X receptor attenuates hepatic injury in a murine model of alcoholic liver disease. Biochem Biophys Res Commun. 3 janv 2014;443(1):68-73.

18. Aranha MM, Cortez-Pinto H, Costa A, Silva IBM, Camilo ME, Moura MC, et al. Bile acid levels are increased in the liver of patients with steatohepatitis. Eur J Gastroenterol Hepatol. juin 2008;20(6):519-25.

19. Williams GD, Proudfit AH, Quinn EA, Campbell KE. Variations in quantity-frequency measures of alcohol consumption from a general population survey. Addiction. avr 1994;89(4):413-20.

20. Alcoholic liver disease: morphological manifestations. Review by an international group. Lancet. 28 mars 1981;1(8222):707-11.

21. Crabb DW, Bataller R, Chalasani NP, Kamath PS, Lucey M, Mathurin P, et al. Standard Definitions and Common Data Elements for Clinical Trials in Patients With Alcoholic Hepatitis: Recommendation From the NIAAA Alcoholic Hepatitis Consortia. Gastroenterology. avr 2016;150(4):785-90.

22. Humbert L, Maubert MA, Wolf C, Duboc H, Mahé M, Farabos D, et al. Bile acid profiling in human biological samples: comparison of extraction procedures and application to normal and cholestatic patients. J Chromatogr B Analyt Technol Biomed Life Sci. 15 juin 2012;899:135-45.

23. Ferrere G, Wrzosek L, Cailleux F, Turpin W, Puchois V, Spatz M, et al. Fecal microbiota manipulation prevents dysbiosis and alcohol-induced liver injury in mice. J Hepatol. avr 2017;66(4):806-15.

24. Langille MGI, Zaneveld J, Caporaso JG, McDonald D, Knights D, Reyes JA, et al. Predictive functional profiling of microbial communities using 16S rRNA marker gene sequences. Nat Biotechnol. sept 2013;31(9):814-21.

25. Segata N, Izard J, Waldron L, Gevers D, Miropolsky L, Garrett WS, et al. Metagenomic biomarker discovery and explanation. Genome Biol. 24 juin 2011;12(6):R60.

26. Xia J, Wishart DS. Using MetaboAnalyst 3.0 for Comprehensive Metabolomics Data Analysis. Curr Protoc Bioinformatics. 7 sept 2016;55:14.10.1-14.10.91. 
641

642

643

644

645

646

647

648

649

650

651

652

653

654

655

656

657

658

659

660

661

662

663

664

665

666

667

668

669

670

671

672

673

674

675

676

677

678

679
27. Grander C, Adolph TE, Wieser V, Lowe P, Wrzosek L, Gyongyosi B, et al. Recovery of ethanol-induced Akkermansia muciniphila depletion ameliorates alcoholic liver disease. Gut. mai 2018;67(5):891-901.

28. Mutlu EA, Gillevet PM, Rangwala H, Sikaroodi M, Naqvi A, Engen PA, et al. Colonic microbiome is altered in alcoholism. Am J Physiol Gastrointest Liver Physiol. 1 mai 2012;302(9):G966-978.

29. Lowe PP, Gyongyosi B, Satishchandran A, Iracheta-Vellve A, Ambade A, Kodys K, et al. Alcohol-related changes in the intestinal microbiome influence neutrophil infiltration, inflammation and steatosis in early alcoholic hepatitis in mice. PLoS ONE. 2017;12(3):e0174544.

30. Del Chierico F, Nobili V, Vernocchi P, Russo A, Stefanis CD, Gnani D, et al. Gut microbiota profiling of pediatric nonalcoholic fatty liver disease and obese patients unveiled by an integrated meta-omics-based approach. Hepatology. févr 2017;65(2):451-64.

31. Chen P, Miyamoto Y, Mazagova M, Lee K-C, Eckmann L, Schnabl B. Microbiota Protects Mice Against Acute Alcohol-Induced Liver Injury. Alcohol Clin Exp Res. déc 2015;39(12):2313-23.

32. Chen Y, Yang F, Lu H, Wang B, Chen Y, Lei D, et al. Characterization of fecal microbial communities in patients with liver cirrhosis. Hepatology. août 2011;54(2):562-72.

33. Zempleni J, Wijeratne SSK, Hassan YI. Biotin. Biofactors. févr 2009;35(1):36-46.

34. Subramanya SB, Subramanian VS, Kumar JS, Hoiness R, Said HM. Inhibition of intestinal biotin absorption by chronic alcohol feeding: cellular and molecular mechanisms. Am J Physiol Gastrointest Liver Physiol. mars 2011;300(3):G494-501.

35. Lee TD, Sadda MR, Mendler MH, Bottiglieri T, Kanel G, Mato JM, et al. Abnormal hepatic methionine and glutathione metabolism in patients with alcoholic hepatitis. Alcohol Clin Exp Res. janv 2004;28(1):173-81.

36. Tanaka H, Hashiba H, Kok J, Mierau I. Bile salt hydrolase of Bifidobacterium longumbiochemical and genetic characterization. Appl Environ Microbiol. juin 2000;66(6):2502-12.

37. Attili AF, Angelico M, Cantafora A, Alvaro D, Capocaccia L. Bile acid-induced liver toxicity: relation to the hydrophobic-hydrophilic balance of bile acids. Med Hypotheses. janv 1986;19(1):57-69.

38. Hillaire S, Ballet F, Franco D, Setchell KD, Poupon R. Effects of ursodeoxycholic acid and chenodeoxycholic acid on human hepatocytes in primary culture. Hepatology. juill 1995;22(1):82-7.

39. Puri P, Daita K, Joyce A, Mirshahi F, Santhekadur PK, Cazanave S, et al. The presence and severity of nonalcoholic steatohepatitis is associated with specific changes in circulating bile acids. Hepatology. 11 juill 2017; 
680

681

682

683

684

685

686

687

688

689

690

691

692

693

694

695

696

697

698

699

700

701

702

703

704

705

706

707

708

709

710

711

712

713

714

715

716
40. Nilsson L-M, Sjövall J, Strom S, Bodin K, Nowak G, Einarsson C, et al. Ethanol stimulates bile acid formation in primary human hepatocytes. Biochem Biophys Res Commun. 28 déc 2007;364(4):743-7.

41. Schrumpf E, Kummen M, Valestrand L, Greiner TU, Holm K, Arulampalam V, et al. The gut microbiota contributes to a mouse model of spontaneous bile duct inflammation. J Hepatol. févr 2017;66(2):382-9.

42. Zweers SJ, Vries EM, Lenicek M, Tolenaars D, Waart DR, Koelfat KVK, et al. Prolonged fibroblast growth factor 19 response in patients with primary sclerosing cholangitis after an oral chenodeoxycholic acid challenge. Hepatol Int. janv 2017;11(1):132-40.

43. Song K-H, Li T, Owsley E, Strom S, Chiang JYL. Bile acids activate fibroblast growth factor 19 signaling in human hepatocytes to inhibit cholesterol $7 \alpha$-hydroxylase gene expression. Hepatology. janv 2009;49(1):297-305.

44. Wunsch E, Milkiewicz M, Wasik U, Trottier J, Kempińska-Podhorodecka A, Elias E, et al. Expression of hepatic Fibroblast Growth Factor 19 is enhanced in Primary Biliary Cirrhosis and correlates with severity of the disease. Sci Rep. 21 août 2015;5:13462.

45. Colell A, Coll O, García-Ruiz C, París R, Tiribelli C, Kaplowitz N, et al. Tauroursodeoxycholic acid protects hepatocytes from ethanol-fed rats against tumor necrosis factor-induced cell death by replenishing mitochondrial glutathione. Hepatology. nov 2001;34(5):964-71.

46. Pelletier G, Roulot D, Davion T, Masliah C, Causse X, Oberti F, et al. A randomized controlled trial of ursodeoxycholic acid in patients with alcohol-induced cirrhosis and jaundice. Hepatology. avr 2003;37(4):887-92.

47. Lepercq P, Hermier D, David O, Michelin R, Gibard C, Beguet F, et al. Increasing ursodeoxycholic acid in the enterohepatic circulation of pigs through the administration of living bacteria. Br J Nutr. avr 2005;93(4):457-69.

48. Van Eldere J, Celis P, De Pauw G, Lesaffre E, Eyssen H. Tauroconjugation of cholic acid stimulates 7 alpha-dehydroxylation by fecal bacteria. Appl Environ Microbiol. févr 1996;62(2):656-61.

49. Raimondi F, Santoro P, Barone MV, Pappacoda S, Barretta ML, Nanayakkara M, et al. Bile acids modulate tight junction structure and barrier function of Caco-2 monolayers via EGFR activation. Am J Physiol Gastrointest Liver Physiol. avr 2008;294(4):G906913.

50. Floch MH, Binder HJ, Filburn B, Gershengoren W. The effect of bile acids on intestinal microflora. Am J Clin Nutr. déc 1972;25(12):1418-26.

51. Kurdi P, Kawanishi K, Mizutani K, Yokota A. Mechanism of growth inhibition by free bile acids in lactobacilli and bifidobacteria. J Bacteriol. mars 2006;188(5):1979-86. 
717 52. Islam KBMS, Fukiya S, Hagio M, Fujii N, Ishizuka S, Ooka T, et al. Bile acid is a host factor that regulates the composition of the cecal microbiota in rats. Gastroenterology. nov 2011;141(5):1773-81.

53. Mathurin P, Deng QG, Keshavarzian A, Choudhary S, Holmes EW, Tsukamoto H. Exacerbation of alcoholic liver injury by enteral endotoxin in rats. Hepatology. nov 2000;32(5):1008-17.

54. Bajaj JS, Cox IJ, Betrapally NS, Heuman DM, Schubert ML, Ratneswaran M, et al. Systems biology analysis of omeprazole therapy in cirrhosis demonstrates significant shifts in gut microbiota composition and function. Am J Physiol Gastrointest Liver Physiol. 15 nov 2014;307(10):G951-957. 
Table 1: Patient's characteristics

\begin{tabular}{|c|c|c|c|c|}
\hline & \multicolumn{2}{|c|}{ Without alcoholic cirrhosis } & \multicolumn{2}{|c|}{ Alcoholic cirrhosis } \\
\hline & noCir_noAH & noCir_AH & Cir_noAH & Cir_sAH \\
\hline & $(n=61)$ & $(n=\overline{13})$ & $(n=17)$ & $(n=17)$ \\
\hline Age (years) & $51 \pm 8.5$ & $46 \pm 8.1$ & $58 \pm 9.8$ & $56 \pm 12.3$ \\
\hline Sex: male/female (\%) & $50 / 10(83 / 17)$ & $11 / 2(85 / 15)$ & $15 / 3(83 / 17)$ & $14 / 4(78 / 22)$ \\
\hline BMI (kg/m2) & $23.4[16.4-31.2]$ & $23.9[20-41.2]$ & $23.7[15.9-31.2]$ & 24.2 [19.4-39] \\
\hline Alcohol intake (g/day) & $150[50-500]$ & $200[60-400]$ & $140[50-360]$ & $80[50-240]^{*}$ \\
\hline Alcohol time (years) & $15[0.5-40]$ & $10[1-40]$ & $22.5[3-50]$ & $20[6-40]$ \\
\hline Smoking (\%) & $48(81)$ & $9(70)$ & $13(72)$ & $13(72)$ \\
\hline PPIs use (\%) & $5(8)$ & $1(8)$ & $7(41)$ & $7(41)$ \\
\hline Diabetes & $5(8)$ & $1(8)$ & $4(22)$ & $2(11)$ \\
\hline AST (U/L) & $47[15-240]$ & $175[58-511]^{\star * *}$ & 64 [38-252] & $88[18-2657]^{\star}$ \\
\hline $\operatorname{ALT}(\mathrm{U} / \mathrm{L})$ & $40.5[8-224]$ & $59[32-217]^{\star}$ & $40.5[11-143]$ & $41.5[19-481]$ \\
\hline Total bilirubin $(\mu \mathrm{mol} / \mathrm{L})$ & $12[6-34]$ & $22[10-186]^{* *}$ & $34.5[4-110]$ & $143[44-751]^{\star \star \star}$ \\
\hline GGT (U/L) & $141[18-1641]$ & $928[79-3970]^{\star *}$ & $382[58-3923]$ & $185.5[45-990]$ \\
\hline Platelets $\left(\times 10^{\wedge} 9 / \mathrm{L}\right)$ & $189.5[36-455]$ & $193[71-459]$ & $98.5[53-378]$ & $94[33-475]$ \\
\hline Prothrombine Time (\%) & $100[67-100]$ & 91 [79-100] & $66[25-100]$ & $35[25-64]^{\star * *}$ \\
\hline Glucose (mmol/L) & $4.9[3.8-11.8]$ & $4.8[4.3-7.5]$ & $5.2[4.4-13.6]$ & $5.2[4.4-8.9]$ \\
\hline Albumin (g/L) & $37.8[27.6-46.7]$ & $39.5[23.1-47.5]$ & $36.1[21.5-40.8]$ & $30[22.3-36]^{\star \star *}$ \\
\hline Creatinin $(\mu \mathrm{mol} / \mathrm{L})$ & $74[50-107]$ & $73[57-118]$ & $71[52-130]$ & $73[57-207]$ \\
\hline $\mathrm{CRP}(\mathrm{mg} / \mathrm{L})$ & $5[5-54]$ & $8[5-91]^{\star}$ & $5[5-34]$ & $22[5-127]^{\star *}$ \\
\hline Maddrey Score & $1.15[0.35-30.9]$ & $5.5[0.59-16.5]$ & $19[0.2-60]$ & $48.9[32-101]^{\star * *}$ \\
\hline FGF-19 (pg/mL) & $86[0-600]$ & $36.18[4.8-1258]$ & 66 [16-541] & $166[24-2450]$ \\
\hline MELD Score & & & $13.7 \pm 7.2$ & $24.33 \pm 6.8^{* \star *}$ \\
\hline Liver Biopsy (\%) & $24(39)$ & $11(85)^{*}$ & $12(71)$ & $17(100)^{*}$ \\
\hline
\end{tabular}

The data are expressed as the mean \pm SD for continuous variables with a normal distribution, median and $\min$ and max for data with a non-normal distribution, and $n(\%)$ for discrete variables. Comparisons between noCir and $\mathrm{AH}$ patients, and alcoholic cirrhosis (Cir) and severe alcoholic hepatitis (sAH) patients in Mann-Whitney tests or independent $t$-tests for continuous data and $x^{2}$ tests or Fisher's exact tests for discrete data. ${ }^{*} p<0.05 ;{ }^{* *} p<0.01 ;{ }^{* * *} p<0.001$. BMI, body mass index; PPIs, proton-pump inhibitors, AST, alanine aminotransferase; ALT, aspartate aminotransferase; GGT, gammaglutamyltransferase; CRP, Creactive protein; MELD, Model for End-stage Liver Disease, FGF-19, fibroblast growth factor-19. 


\section{$730 \quad$ Figure legends}

731 Figure 1: Intestinal microbiota profiles and its metabolic functions. (A)

732 Weighted UniFrac distances (quantitative method reflecting the structure of the

733 intestinal microbiota) and (B) Unweighted UniFrac distances (qualitative method

734 reflecting the composition of the intestinal microbiota) showing a difference in the

735 structure of the intestinal microbiota only between Cir_sAH patients (blue) and

736 Cir_noAH patients (red, $p<0.05$ for Weighted UniFrac distances). Each point

737 represents a subject and the distance between the points is proportional to the

738 similarity of the intestinal microbiota. Cladogram showing the taxa with the largest

739 differences in abundance between (C) Cir_sAH patients (green) and Cir_noAH

740 patients (red) and (D) noCir_AH patients (red) and noCir_noAH patients. The size of

741 the circle in the cladogram plot is proportional to bacterial abundance. From inside to

742 outside, the circles represent phylum, class, order, family, and genus. Only taxa with

743 a LDA score $>2$ and $p<0.05$, determined by the Wilcoxon signed rank test, are

744 shown. (E) LDA Effect Size (LEfSe) for the predicted metagenome metabolic

745 pathways (KEGG modules) increased in Cir_sAH (green) and Cir_noAH patients

746 (red) (LDA score $>2.0, p<0.05$ determined by the Wilcoxon signed rank test).

747 Figure 2: Plasma bile acids profiles in patients with alcoholic liver disease. (A)

748 PCA ordination plot with $95 \%$ confidence ellipse for all plasma bile acids s in cir_sAH

749 and cir_noAH patients showing clustering of patients according to the liver

750 complication. The first two components of the PCA explained $64 \%$ of the total

751 variance (component $1=42.4 \%$; component $2=21.5 \%$ ). (B) Total plasma bile acids

752 , primary, total conjugated, glyco-conjugated and tauro-conjugated levels of plasma

753 bile acids . (C) and (D) plasma bile acids composition (\% of total plasma bile acids

754 ). (E) Individual plasma bile acids levels. ${ }^{*} p<0.05$, ${ }^{* *} p<0.01$. CA: cholic acid; 
755 CDCA: chenodeoxycholic acid; DCA: deoxycholic acid; LCA: lithocholic acid; GCA, 756 glycocholic acid: GCDCA: glycochenodeoxycholic acid; GDCA: glycodeoxycholic 757 acid; GLCA: glycolithocholic acid; GUDCA: glycoursodeoxycholic acid; sAH: severe 758 alcoholic hepatitis; TCA: taurocholic acid; TCDCA: taurochenodeoxycholic acid; 759 TDCA: taurodeoxycholic acid; TLCA: taurolithocholic acid; TUDCA: 760 tauroursodeoxycholic acid; UDCA: ursodeoxycholic acid; _3s: sulfated forms.

761 Figure 3: Specificity of the plasma bile acids profile depending on alcoholic762 induced liver inflammation. (A) PLS-DA score plot of plasma bile acids 763 concentrations in Cir_sAH vs. Cir_noAH patients with the 95\% confidence ellipse 764 showing a significant difference between the two groups $(\mathrm{R} 2=0.6, \mathrm{Q} 2=0.4, \mathrm{p}=$ 765 0.001). (B) Variable importance in projection (VIP) of PLS-DA showing the plasma 766 bile acids that discriminate Cir_sAH from Cir_noAH patients (VIP score >1). The 767 colored boxes on the right indicate the relative concentrations of the corresponding 768 plasma bile acids in each group.

769 Figure 4: Faecal bile acids profiles in alcoholic liver disease. (A) PCA ordination 770 plot with $95 \%$ confidence ellipse for all faecal bile acids showing clustering of patients 771 according to the liver complication. The first two components of the PCA explained $77257 \%$ of the total variance (component $1=39.3 \%$; component $2=17.9 \%$ ). (B) Total 773 faecal bile acids, total unconjugated, secondary and secondary unconjugated levels 774 of faecal bile acids. (C) and (D) faecal bile acids composition (\% of total faecal bile 775 acids). (E) Individual faecal bile acids levels. ${ }^{*} p<0.05,{ }^{* *} p<0.01$. CA: cholic acid; 776 CDCA: chenodeoxycholic acid; DCA: deoxycholic acid; LCA: lithocholic acid; GCA: 777 glycocholic acid; GCDCA: glycochenodeoxycholic acid; GDCA: glycodeoxycholic 778 acid; GLCA: glycolithocholic acid; GUDCA: glycoursodeoxycholic acid; sAH: severe 779 alcoholic hepatitis; TCA: taurocholic acid; TCDCA: taurochenodeoxycholic acid; 
780 TDCA: taurodeoxycholic acid; TLCA: taurolithocholic acid; TUDCA:

781 tauroursodeoxycholic acid; UDCA: ursodeoxycholic acid; _3s: sulfated forms.

782 Figure 5: Specificity of faecal bile acids profiles depending on alcoholic783 induced liver inflammation. (A) PLS-DA score plot of faecal bile acids 784 concentrations of Cir_sAH vs. Cir_noAH patients with 95\% confidence ellipse 785 showing a significant difference between the two groups $(R 2=0.8, Q 2=0.6, p=$ 786 0.001). (B) Variable importance in projection (VIP) of PLS-DA showing the faecal bile 787 acids that discriminate Cir_sAH from Cir_noAH patients (VIP score >1). The colored 788 boxes on the right indicate the relative concentrations of the corresponding faecal 789 bile acids in each group.

790 Figure 6: Heatmap representation of the Spearman's $r$ correlation coefficient 791 between bacterial taxa (phylum and genus level) and bile acids profiles in plasma of 792 Cir_noAH $(\mathbf{A})$ and Cir_sAH (B) patients and feces of Cir_noAH (C) and Cir_sAH (D). 793 Only the bacteria for which at least one significant correlation with bile acids was 794 found are displayed ( $p$, phyla; g, genus). CA: cholic acid; CDCA: chenodeoxycholic 795 acid; DCA: deoxycholic acid; LCA: lithocholic acid; GCA: glycocholic acid; GCDCA: 796 glycochenodeoxycholic acid; GDCA: glycodeoxycholic acid; GLCA: glycolithocholic 797 acid; GUDCA: glycoursodeoxycholic acid; TCA: taurocholic acid; TCDCA: 798 taurochenodeoxycholic acid; TDCA: taurodeoxycholic acid; TLCA: taurolithocholic 799 acid; TUDCA: tauroursodeoxycholic acid; UDCA: ursodeoxycholic acid; A: 800 Actinobacteria, B: Bacteroidetes, F: Fusobacteria; P: Proteobacteria. ${ }^{*}$ Adjusted $p$ 801 value < 0.05 . Red: negative correlation, blue: positive correlation. 
Figure 1


C
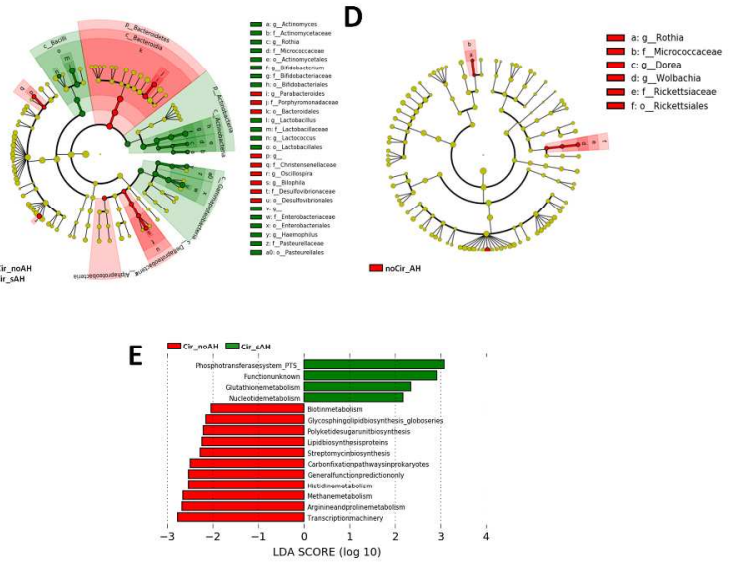

Figure 1: Intestinal microbiota profiles and its metabolic functions. (A) Weighted UniFrac distances (quantitative method reflecting the structure of the intestinal microbiota) and (B) Unweighted UniFrac distances (qualitative method reflecting the composition of the intestinal microbiota) showing a difference in the structure of the intestinal microbiota only between Cir_sAH patients (blue) and Cir_noAH patients (red, p $<0.05$ for Weighted UniFrac distances). Each point represents a subject and the distance between the points is proportional to the similarity of the intestinal microbiota. Cladogram showing the taxa with the largest differences in abundance between (C) Cir_sAH patients (green) and Cir_noAH patients (red) and (D) noCir_AH patients (red) and noCir_noAH patients. The size of the circle in the cladogram plot is proportional

to bacterial abundance. From inside to outside, the circles represent phylum, class, order, family, and genus. Only taxa with a LDA score $>2$ and $p<0.05$, determined by the Wilcoxon signed rank test, are shown. (E) LDA Effect Size (LEfSe) for the predicted metagenome metabolic pathways (KEGG modules) increased in Cir_sAH (green) and Cir_noAH patients (red) (LDA score $>2.0, \mathrm{p}<0.05$ determined by the Wilcoxon signed rank test).

$254 \times 190 \mathrm{~mm}(300 \times 300 \mathrm{DPI})$ 
Figure 2

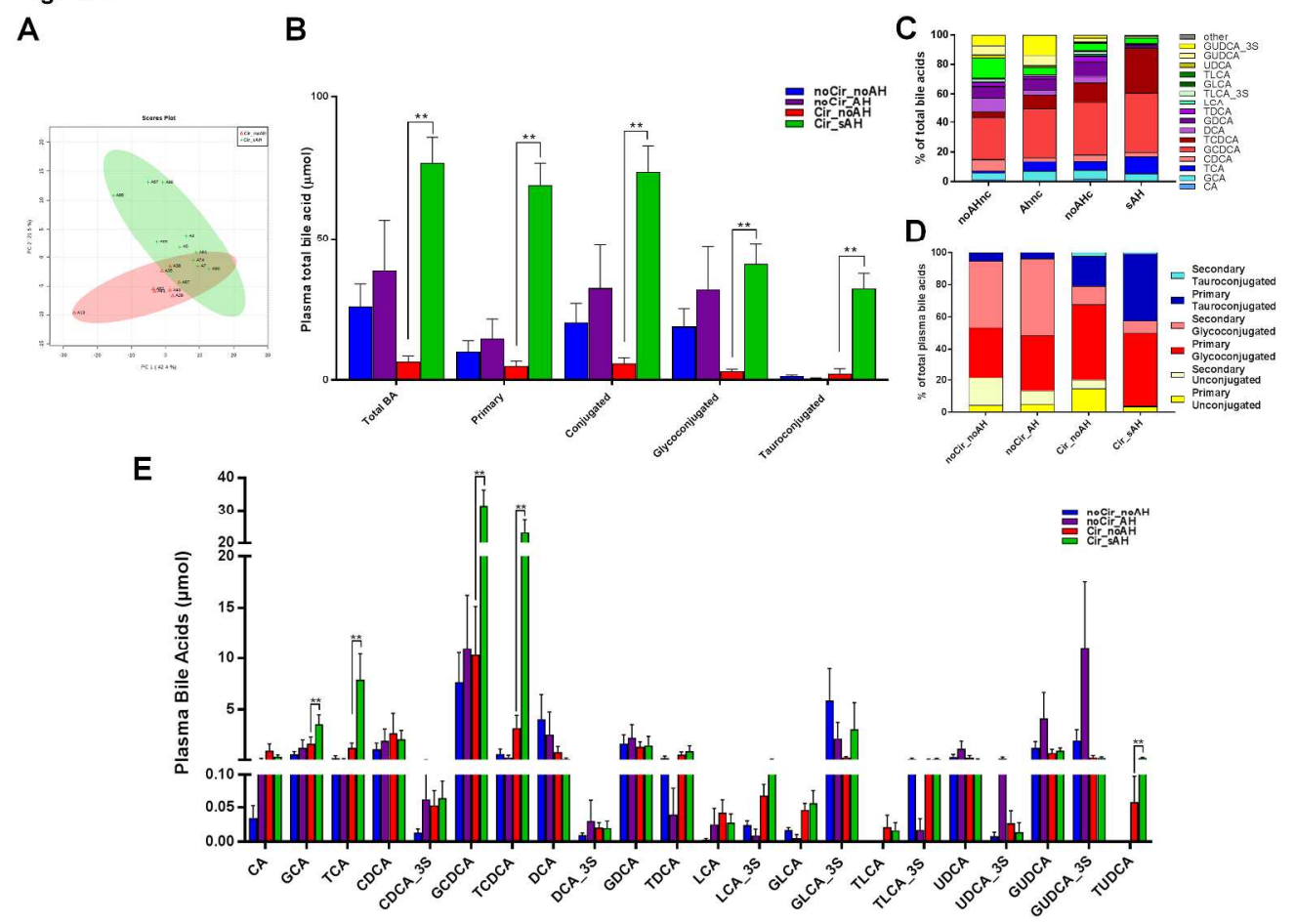

Figure 2: Plasma bile acids profiles in patients with alcoholic liver disease. (A) PCA ordination plot with $95 \%$ confidence ellipse for all plasma bile acids s in cir_sAH and cir_noAH patients showing clustering of patients according to the liver complication. The first two components of the PCA explained $64 \%$ of the total variance (component $1=42.4 \%$; component $2=21.5 \%$ ). (B) Total plasma bile acids , primary, total conjugated, glyco-conjugated and tauro-conjugated levels of plasma bile acids. (C) and (D) plasma bile acids composition ( $\%$ of total plasma bile acids ). (E) Individual plasma bile acids levels. $* p<0.05, * * p$ < 0.01. CA: cholic acid; CDCA: chenodeoxycholic acid; DCA: deoxycholic acid; LCA: lithocholic acid; GCA, glycocholic acid: GCDCA: glycochenodeoxycholic acid; GDCA: glycodeoxycholic acid; GLCA: glycolithocholic acid; GUDCA: glycoursodeoxycholic acid; sAH: severe alcoholic hepatitis; TCA: taurocholic acid; TCDCA: taurochenodeoxycholic acid; TDCA: taurodeoxycholic acid; TLCA: taurolithocholic acid; TUDCA: tauroursodeoxycholic acid; UDCA: ursodeoxycholic acid; _3s: sulfated forms. 
Figure 3: Specificity of the plasma bile acids profile depending on alcoholic-induced liver inflammation. (A) PLS-DA score plot of plasma bile acids concentrations in Cir_sAH vs. Cir_noAH patients with the 95\% confidence ellipse showing a significant difference between the two groups $(R 2=0.6, Q 2=0.4, p=0.001)$.

(B) Variable importance in projection (VIP) of PLS-DA showing the plasma bile acids that discriminate Cir_sAH from Cir_noAH patients (VIP score $>1$ ). The colored boxes on the right indicate the relative concentrations of the corresponding plasma bile acids in each group. 
Figure 4

A

B

Figure 4: Faecal bile acids profiles in alcoholic liver disease. (A) PCA ordination plot with $95 \%$ confidence ellipse for all faecal bile acids showing clustering of patients according to the liver complication. The first two components of the PCA explained $57 \%$ of the total variance (component $1=39.3 \%$; component $2=$ $17.9 \%$ ). (B) Total faecal bile acids, total unconjugated, secondary and secondary unconjugated levels of faecal bile acids. (C) and (D) faecal bile acids composition (\% of total faecal bile acids). (E) Individual faecal bile acids levels. $* p<0.05, * * p<0.01$. CA: cholic acid; CDCA: chenodeoxycholic acid; DCA: deoxycholic acid; LCA: lithocholic acid; GCA: glycocholic acid; GCDCA: glycochenodeoxycholic acid; GDCA: glycodeoxycholic acid; GLCA: glycolithocholic acid; GUDCA: glycoursodeoxycholic acid; sAH: severe alcoholic hepatitis; TCA: taurocholic acid; TCDCA: taurochenodeoxycholic acid; TDCA: taurodeoxycholic acid; TLCA: taurolithocholic acid; TUDCA: tauroursodeoxycholic acid; UDCA: ursodeoxycholic acid; _3s: sulfated forms. 


\section{Figure 5}

Figure 5: Specificity of faecal bile acids profiles depending on alcoholic-induced liver inflammation. (A) PLSDA score plot of faecal bile acids concentrations of Cir_sAH vs. Cir_noAH patients with $95 \%$ confidence ellipse showing a significant difference between the two groups $(\bar{R} 2=0.8, Q 2=0.6, p=0.001)$. (B) Variable importance in projection (VIP) of PLS-DA showing the faecal bile acids that discriminate Cir_sAH from Cir_noAH patients (VIP score $>1$ ). The colored boxes on the right indicate the relative concentrations of the corresponding faecal bile acids in each group.

$254 \times 190 \mathrm{~mm}(300 \times 300 \mathrm{DPI})$ 
Figure 6

A

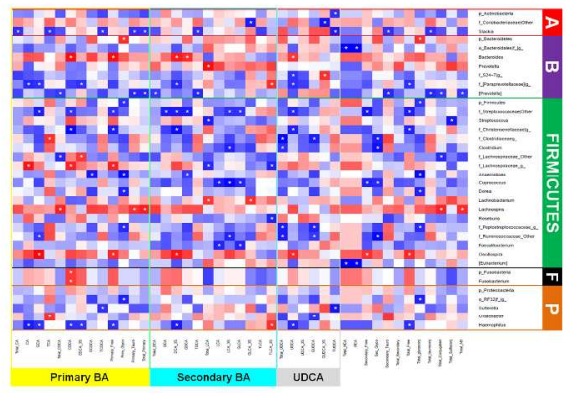

C

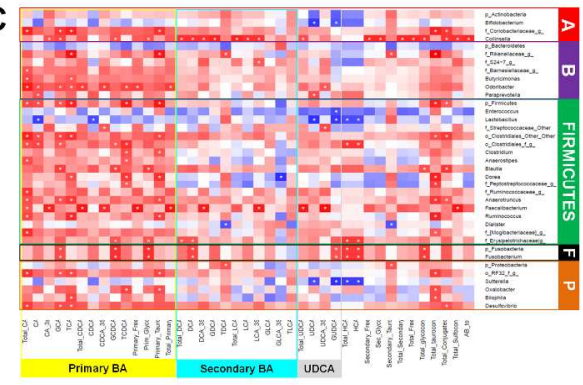

B



D

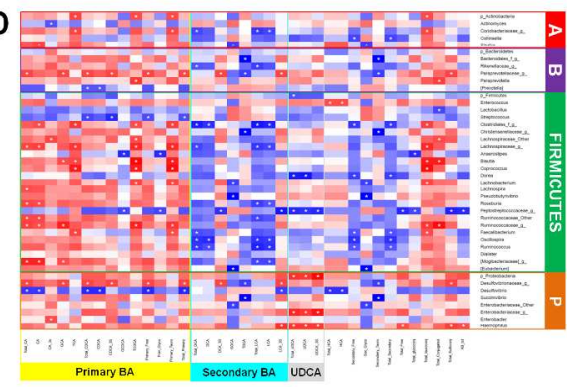

Figure 6: Heatmap representation of the Spearman's $r$ correlation coefficient between bacterial taxa (phylum and genus level) and bile acids profiles in plasma of Cir_noAH (A) and Cir_sAH (B) patients and feces of Cir_noAH (C) and Cir_sAH (D). Only the bacteria for which at least one significant correlation with bile acids was found are displayed ( $p$, phyla; g, genus). CA: cholic acid; CDCA: chenodeoxycholic acid; DCA: deoxycholic acid; LCA: lithocholic acid; GCA: glycocholic acid; GCDCA: glycochenodeoxycholic acid; GDCA: glycodeoxycholic acid; GLCA: glycolithocholic acid; GUDCA: glycoursodeoxycholic acid; TCA: taurocholic acid; TCDCA: taurochenodeoxycholic acid; TDCA: taurodeoxycholic acid; TLCA: taurolithocholic acid; TUDCA: tauroursodeoxycholic acid; UDCA: ursodeoxycholic acid; A: Actinobacteria, B: Bacteroidetes, F: Fusobacteria; P: Proteobacteria. *Adjusted p value $<0.05$. Red: negative correlation, blue: positive correlation. 
Title: Bile acids homeostasis and intestinal dysbiosis in alcoholic hepatitis

Running Title: Bile acid, microbiota and alcoholic hepatitis

Dragos Ciocan ${ }^{1}$, Cosmin Sebastian Voican ${ }^{1,2}$, Laura Wrzosek ${ }^{1,2}$, Cindy Hugot ${ }^{1}$, Dominique Rainteau $^{3,4,5,6}$, Lydie Humbert ${ }^{3,4,5}$, Anne-Marie Cassard ${ }^{1}$, and Gabriel Perlemuter $^{1,2}$

${ }^{1}$ Inflammation Chimiokines et Immunopathologie, DHU Hepatinov, Faculté de Médecine - Université Paris-Sud, INSERM, Université Paris-Saclay, 92140, Clamart, France;

${ }^{2} \mathrm{AP}-\mathrm{HP}$, Hepatogastroenterology and Nutrition, Hôpital Antoine-Béclère, Clamart, France;

${ }^{3}$ Sorbonne Universités, UPMC Université Paris 6, Paris, France;

${ }^{4}$ Inflammation-Immunopathology-Biotherapy Department (DHU i2B), INSERM-ERL 1157, Paris, France;

${ }^{5}$ UMR 7203 Laboratoire des Biomolécules, UPMC/CNRS/ENS, Paris, France;

${ }^{6}$ Département PM2 Plateforme de Métabolomique, APHP, Hôpital Saint Antoine, Peptidomique et dosage de Médicaments, Paris, France;

\section{Co-Corresponding authors:}

Prof. Gabriel Perlemuter, Service d'Hépato-Gastroentérologie et Nutrition, Hôpital Antoine-Béclère, 157 rue de la Porte de Trivaux, Clamart cedex F-92141, France; gabriel.perlemuter@aphp.fr.

Dr Anne-Marie Cassard, INSERM U996, 32 rue des Carnets, Clamart, F-92140, France; cassard.doulcier@u-psud.fr 
Supplementary Table 1: Differences in intestinal microbiota at the genus level between patients with alcoholic cirrhosis, with (Cir_sAH ) and without severe alcoholic hepatitis (Cir_noAH).

\begin{tabular}{|c|c|c|c|c|c|c|c|c|c|}
\hline \multirow[t]{2}{*}{ Phyla } & \multirow[t]{2}{*}{ Family } & \multirow[t]{2}{*}{ Genus } & \multirow{2}{*}{$\begin{array}{l}\text { Increased } \\
\text { in }\end{array}$} & \multicolumn{2}{|c|}{ Relative abundance } & \multicolumn{2}{|c|}{ Mann Whitney } & \multicolumn{2}{|c|}{ LEfSe } \\
\hline & & & & Cir_noAH & Cir_sAH & $p$ & FDR & LDA score & $p$ \\
\hline \multirow{3}{*}{ Actinobacteria } & Actinomycetaceae & Actinomyces & Cir_sAH & $18^{*} 10^{-5}$ & $79 * 10^{-5}$ & 0.03 & 0.23 & 3.06 & 0.03 \\
\hline & Micrococcaceae & Rothia & Cir_sAH & $1 * 10^{-5}$ & $22 * 10^{-5}$ & 0.01 & 0.20 & 3.29 & 0.01 \\
\hline & Bifidobacteriaceae & Bifidobacterium & Cir_sAH & $1200 * 10^{-5}$ & $8373^{*} 10^{-5}$ & 0.02 & 0.23 & 3.84 & 0.02 \\
\hline Bacteroidetes & Porphyromonadaceae & Parabacteroides & Cir_noAH & $1665^{*} 10^{-5}$ & $555^{*} 10^{-5}$ & 0.03 & 0.23 & 3.14 & 0.03 \\
\hline \multirow{5}{*}{ Firmicutes } & Streptococcaceae & Lactococcus & Cir_sAH & $68 * 10^{-5}$ & $79 * 10^{-5}$ & 0.04 & 0.27 & 2.87 & 0.04 \\
\hline & Christensenellaceae & $g_{-}$ & Cir_noAH & $172^{*} 10^{-5}$ & $3 * 10^{-5}$ & 0.03 & 0.23 & 2.73 & 0.03 \\
\hline & Ruminococcaceae & Oscillospira & Cir_noAH & $1247^{*} 10^{-5}$ & $421 * 10^{-5}$ & 0.00 & 0.14 & 3.00 & $<0.01$ \\
\hline & Lactobacillaceae & Lactobacillus & Cir_sAH & $1294^{*} 10^{-5}$ & $12397^{*} 10^{-5}$ & 0.00 & 0.14 & 4.09 & $<0.01$ \\
\hline & Pasteurellaceae & Haemophilus & Cir_sAH & $165^{\star} 10^{-5}$ & $503^{*} 10^{-5}$ & 0.01 & 0.22 & 2.88 & 0.01 \\
\hline \multirow[t]{2}{*}{ Proteobacteria } & Desulfovibrionaceae & Bilophila & Cir_noAH & $217^{*} 10^{-5}$ & $26^{*} 10^{-5}$ & 0.01 & 0.20 & 2.97 & 0.01 \\
\hline & Enterobacteriaceae & $g_{-}$ & Cir_sAH & $3601 * 10^{-5}$ & $8156^{*} 10^{-5}$ & 0.02 & 0.23 & 3.79 & 0.02 \\
\hline
\end{tabular}


Supplementary Table 2: Plasma bile acids concentrations between groups.

\begin{tabular}{|c|c|c|c|c|c|c|c|c|c|c|c|c|}
\hline \multirow{3}{*}{$\begin{array}{l}\text { Plasma Bile Acids } \\
\qquad(n=56)\end{array}$} & \multicolumn{6}{|c|}{ Without cirrhosis } & \multicolumn{6}{|c|}{ Cirrhosis } \\
\hline & \multicolumn{3}{|c|}{$\begin{array}{c}\text { noCir_noAH } \\
(n=29)\end{array}$} & \multicolumn{3}{|c|}{$\begin{array}{c}\text { noCir_AH } \\
(n=8)\end{array}$} & \multicolumn{3}{|c|}{$\begin{array}{c}\text { Cir_noAH } \\
(\mathrm{n}=8)\end{array}$} & \multicolumn{3}{|c|}{$\begin{array}{l}\text { Cir_sAH } \\
(n=10)\end{array}$} \\
\hline & Median & Min & Max & Median & Min & Max & Median & Min & Max & Median & Min & Max \\
\hline $\mathrm{CA}$ & 0 & 0 & 0.51 & 0.00 & 0 & 0.90 & 0 & 0 & 0.33 & 0.19 & 0 & 1.38 \\
\hline GCA & 0.07 & 0 & 7.10 & 0.26 & 0.002 & 5.36 & 0.36 & 0 & 0.54 & 3.11 & 0 & 9.30 \\
\hline TCA & 0.03 & 0 & 6.44 & 0.01 & 0 & 0.74 & 0.16 & 0 & 2.78 & 4.48 & 0 & 25.46 \\
\hline Total CA & 0.16 & 0 & 13.54 & 0.32 & 0.003 & 6.25 & 0.59 & 0 & 3.05 & 8.46 & 0 & 34.82 \\
\hline CDCA & 0.07 & 0 & 17.95 & 0.03 & 0 & 8.37 & 0.02 & 0 & 0.63 & 1.49 & 0 & 9.15 \\
\hline CDCA_3S & 0 & 0 & 0.16 & 0.00 & 0 & 0.28 & 0.02 & 0 & 0.07 & 0.02 & 0 & 0.22 \\
\hline GCDCA & 1.05 & 0 & 56.08 & 1.49 & 0.01 & 32.55 & 2.49 & 0.002 & 4.10 & 34.89 & 7.36 & 57.37 \\
\hline TCDCA & 0.06 & 0 & 12.81 & 0.05 & 0 & 1.83 & 0.48 & 0 & 9.05 & 21.97 & 5.02 & 45.01 \\
\hline Total CDCA & 1.73 & 0.001 & 68.89 & 1.85 & 0.01 & 42.94 & 4.00 & 0.002 & 11.39 & 61.35 & 12.38 & 81.23 \\
\hline Total Primary & 1.90 & 0.002 & 82.43 & 2.30 & 0.01 & 43.24 & 4.74 & 0.002 & 14.44 & 70.67 & 16.57 & 99.42 \\
\hline DCA & 0.11 & 0 & 67.94 & 0.24 & 0 & 17.99 & 0.16 & 0.001 & 0.55 & 0.01 & 0 & 0.88 \\
\hline DCA_3S & 0 & 0 & 0.07 & 0 & 0 & 0.24 & 0 & 0 & 0.05 & 0 & 0 & 0.10 \\
\hline GDCA & 0.21 & 0 & 23.50 & 0.75 & 0 & 10.59 & 0.50 & 0.002 & 1.19 & 0.16 & 0 & 6.98 \\
\hline TDCA & 0.02 & 0 & 6.30 & 0.00 & 0 & 0.31 & 0.14 & 0 & 2.01 & 0.17 & 0 & 4.51 \\
\hline Total DCA & 0.47 & 0 & 70.94 & 1.15 & 0 & 28.58 & 1.02 & 0.002 & 2.51 & 0.36 & 0 & 12.19 \\
\hline LCA & 0 & 0 & 0.03 & 0 & 0 & 0.19 & 0.02 & 0 & 0.08 & 0.01 & 0 & 0.11 \\
\hline LCA_3S & 0 & 0 & 0.11 & 0 & 0 & 0.07 & 0.06 & 0 & 0.23 & 0.04 & 0 & 0.48 \\
\hline GLCA & 0 & 0 & 0.07 & 0 & 0 & 0.04 & 0.05 & 0 & 0.10 & 0.04 & 0 & 0.17 \\
\hline GLCA_3S & 0.18 & 0 & 74.05 & 0.007 & 0 & 12.83 & 0.27 & 0 & 0.68 & 0.19 & 0 & 26.48 \\
\hline $\mathrm{TLCA}^{-}$ & 0 & 0 & 0 & 0 & 0 & 0 & 0 & 0 & 0.20 & 0 & 0 & 0.10 \\
\hline TLCA_3S & 0 & 0 & 3.49 & 0 & 0 & 0.13 & 0.09 & 0 & 0.66 & 0.11 & 0 & 0.76 \\
\hline Total LCA & 0.29 & 0 & 74.05 & 0.01 & 0 & 13.02 & 0.66 & 0.001 & 1.25 & 0.47 & 0 & 26.48 \\
\hline UDCA & 0.03 & 0 & 7.97 & 0.00 & 0 & 5.94 & 0.01 & 0 & 0.06 & 0.05 & 0 & 0.73 \\
\hline UDCA_3S & 0 & 0 & 0.13 & 0 & 0 & 1.01 & 0 & 0 & 0.13 & 0 & 0 & 0.14 \\
\hline GUDCA & 0.17 & 0 & 13.26 & 0.32 & 0.000 & 20.54 & 0.13 & 0 & 0.27 & 0.76 & 0 & 2.49 \\
\hline GUDCA_3S & 0.08 & 0 & 22.34 & 0.24 & 0 & 49.30 & 0.09 & 0 & 0.15 & 0.13 & 0 & 1.16 \\
\hline TUDCA & 0 & 0 & 0.01 & 0 & 0 & 0.00 & 0 & 0 & 0.02 & 0.26 & 0 & 0.66 \\
\hline Total UDCA & 0.37 & 0 & 42.49 & 0.56 & 0.001 & 56.73 & 0.28 & 0 & 0.44 & 1.30 & 0 & 4.09 \\
\hline Total Secondary & 1.68 & 0.002 & 96.84 & 2.32 & 0.00 & 66.00 & 2.12 & 0.004 & 3.93 & 2.47 & 0.58 & 26.48 \\
\hline Total Unconjugated & 0.64 & 0 & 85.89 & 0.37 & 0 & 26.49 & 0.27 & 0.001 & 1.74 & 1.89 & 0 & 12.02 \\
\hline Total Glycoconjugated & 2.32 & 0.002 & 96.49 & 3.56 & 0.01 & 93.90 & 3.90 & 0.005 & 6.58 & 39.98 & 9.11 & 83.85 \\
\hline Total Tauroconjugated & 0.16 & 0 & 19.25 & 0.36 & 0.003 & 1.83 & 0.90 & 0 & 14.71 & 32.17 & 8.19 & 60.33 \\
\hline Total Sulfoconjugated & 0.50 & 0 & 74.05 & 0.84 & 0.001 & 54.38 & 0.79 & 0.001 & 1.06 & 0.65 & 0.06 & 26.48 \\
\hline Total Conjugated & 2.60 & 0.002 & 100 & 4.26 & 0.01 & 93.98 & 5.89 & 0.005 & 18.19 & 81.77 & 17.30 & 100 \\
\hline Total Bile Acids & 3.60 & 0.003 & 100 & 4.62 & 0.01 & 100 & 6.84 & 0.01 & 18.36 & 84.57 & 17.37 & 100 \\
\hline
\end{tabular}


Supplementary Table 3: Fecal bile acids concentrations between groups.

\begin{tabular}{|c|c|c|c|c|c|c|c|c|c|c|c|c|}
\hline \multirow{3}{*}{$\begin{array}{l}\text { Fecal Bile Acids } \\
\qquad(n=73)\end{array}$} & \multicolumn{6}{|c|}{ Without cirrhosis } & \multicolumn{6}{|c|}{ Cirrhosis } \\
\hline & \multicolumn{3}{|c|}{$\begin{array}{c}\text { noCir_noAH } \\
(n=46)\end{array}$} & \multicolumn{3}{|c|}{$\begin{array}{c}\text { noCir_AH } \\
(n=6)\end{array}$} & \multicolumn{3}{|c|}{$\begin{array}{c}\text { Cir_noAH } \\
(n=10)\end{array}$} & \multicolumn{3}{|c|}{$\begin{array}{c}\text { Cir_sAH } \\
(n=11)\end{array}$} \\
\hline & Median & Min & Max & Median & Min & Max & Median & Min & Max & Median & Min & Max \\
\hline$C A$ & 0.07 & 0 & 11.66 & 0.10 & 0 & 1.02 & 0.03 & 0 & 49.09 & 0.09 & 0.01 & 1.56 \\
\hline CA_3s & 0 & 0 & 0.32 & 0.02 & 0.001 & 0.05 & 0.02 & 0 & 1.14 & 0.001 & 0 & 0.08 \\
\hline $\mathrm{GCA}$ & 0.01 & 0.001 & 3.17 & 0.01 & 0.01 & 0.13 & 0.01 & 0 & 7.62 & 0 & 0 & 0.01 \\
\hline TCA & 0.01 & 0 & 1.90 & 0 & 0 & 0.04 & 0.01 & 0 & 10.07 & 0.01 & 0 & 0.08 \\
\hline Total CA & 0.17 & 0.003 & 12.13 & 0.14 & 0.02 & 1.07 & 0.23 & 0 & 66.82 & 0.10 & 0.01 & 1.58 \\
\hline CDCA & 0.16 & 0.01 & 6.82 & 0.08 & 0.01 & 2.73 & 0.05 & 0.005 & 34.24 & 0.11 & 0.04 & 6.77 \\
\hline CDCA_3S & 0.02 & 0 & 4.16 & 0.07 & 0.003 & 0.36 & 0.32 & 0 & 1.30 & 0.01 & 0 & 0.18 \\
\hline GCDCA & 0.05 & 0 & 4.35 & 0.05 & 0.01 & 0.13 & 0.02 & 0.002 & 5.44 & 0.01 & 0.001 & 0.04 \\
\hline TCDCA & 0.01 & 0 & 0.94 & 0.01 & 0 & 0.03 & 0.01 & 0.001 & 0.05 & 0.02 & 0.004 & 0.03 \\
\hline Total CDCA & 0.45 & 0.05 & 9.61 & 0.33 & 0.11 & 2.93 & 0.64 & 0.01 & 40.34 & 0.31 & 0.05 & 6.84 \\
\hline Total Primary & 0.78 & 0.07 & 20.72 & 0.53 & 0.13 & 4.00 & 0.89 & 0.01 & 107.16 & 0.38 & 0.08 & 7.75 \\
\hline DCA & 3.78 & 0.01 & 24.12 & 5.61 & 0.28 & 22.36 & 3.76 & 0.62 & 25.27 & 0.13 & 0.01 & 2.61 \\
\hline DCA_3S & 0.14 & 0.002 & 22.06 & 0.30 & 0.01 & 4.45 & 0.07 & 0 & 9.86 & 0 & 0 & 1.24 \\
\hline GDCA & 0.02 & 0 & 1.05 & 0.03 & 0 & 0.12 & 0.01 & 0 & 0.10 & 0 & 0 & 0.003 \\
\hline TDCA & 0.01 & 0 & 0.41 & 0.01 & 0 & 0.04 & 0.01 & 0 & 0.08 & 0 & 0 & 0.002 \\
\hline Total DCA & 4.54 & 0.05 & 46.49 & 7.43 & 0.36 & 22.74 & 6.49 & 0.63 & 25.55 & 0.13 & 0.01 & 2.61 \\
\hline LCA & 1.96 & 0.003 & 8.74 & 1.96 & 0.38 & 20.00 & 1.95 & 0.02 & 5.43 & 0.58 & 0.005 & 2.93 \\
\hline LCA_3S & 0.17 & 0 & 18.64 & 0.60 & 0.004 & 7.82 & 0.21 & 0 & 18.31 & 0 & 0 & 0.25 \\
\hline GLCA & 0 & 0 & 0.003 & 0 & 0 & 0.02 & 0.00 & 0 & 0 & 0 & 0 & 0 \\
\hline GLCA_3S & 0 & 0 & 0.06 & 0.01 & 0 & 0.21 & 0.00 & 0 & 0.03 & 0 & 0 & 0 \\
\hline TLCA & 0 & 0 & 0 & 0.00 & 0 & 0.001 & 0.00 & 0 & 0 & 0 & 0 & 0 \\
\hline Total LCA & 2.53 & 0.05 & 22.91 & 2.93 & 0.44 & 28.04 & 2.69 & 0.38 & 20.15 & 0.58 & 0.01 & 2.93 \\
\hline UDCA & 0.10 & 0 & 7.18 & 0.06 & 0 & 1.47 & 0.01 & 0.002 & 2.38 & 0.01 & 0.003 & 1.94 \\
\hline UDCA_3S & 0.04 & 0 & 4.09 & 0.04 & 0 & 0.63 & 0.09 & 0 & 0.90 & 0 & 0 & 0.23 \\
\hline GUDCA & 0 & 0 & 0.15 & 0.01 & 0 & 0.02 & 0 & 0 & 0.11 & 0 & 0 & 0 \\
\hline GUDCA_3S & 0 & 0 & 0.08 & 0 & 0 & 0 & 0 & 0 & 0 & 0 & 0 & 0 \\
\hline TUDCA & 0 & 0 & 0.04 & 0 & 0 & 0.001 & 0 & 0 & 0 & 0 & 0 & 0 \\
\hline Total UDCA & 0.30 & 0.01 & 11.31 & 0.39 & 0.02 & 1.54 & 0.41 & 0.002 & 3.11 & 0.02 & 0.003 & 1.94 \\
\hline Total Secondary & 8.40 & 0.21 & 63.85 & 10.24 & 0.83 & 52.40 & 10.68 & 1.80 & 27.92 & 0.72 & 0.02 & 5.68 \\
\hline Total Unconjugated & 9.33 & 0.40 & 83.97 & 10.54 & 0.93 & 53.27 & 11.17 & 1.80 & 111.58 & 2.30 & 0.19 & 10.97 \\
\hline Total Glycoconjugated & 0.10 & 0.002 & 7.67 & 0.10 & 0.03 & 0.51 & 0.05 & 0.002 & 13.30 & 0.02 & 0.001 & 0.05 \\
\hline Total Tauroconjugated & 0.02 & 0 & 2.86 & 0.02 & 0 & 0.11 & 0.02 & 0.001 & 10.20 & 0.02 & 0.01 & 0.10 \\
\hline Total Sulfoconjugated & 0.46 & 0.01 & 31.88 & 1.23 & 0.05 & 8.36 & 0.71 & 0 & 25.13 & 0.04 & 0 & 1.99 \\
\hline Total Conjugated & 0.12 & 0.002 & 10.54 & 0.16 & 0.03 & 0.53 & 0.08 & 0.02 & 23.50 & 0.03 & 0.02 & 0.12 \\
\hline Total BA & 9.87 & 1.21 & 84.57 & 10.77 & 0.96 & 53.80 & 11.32 & 1.82 & 135.08 & 2.36 & 0.31 & 11.03 \\
\hline
\end{tabular}




\section{Supplemental Figures}
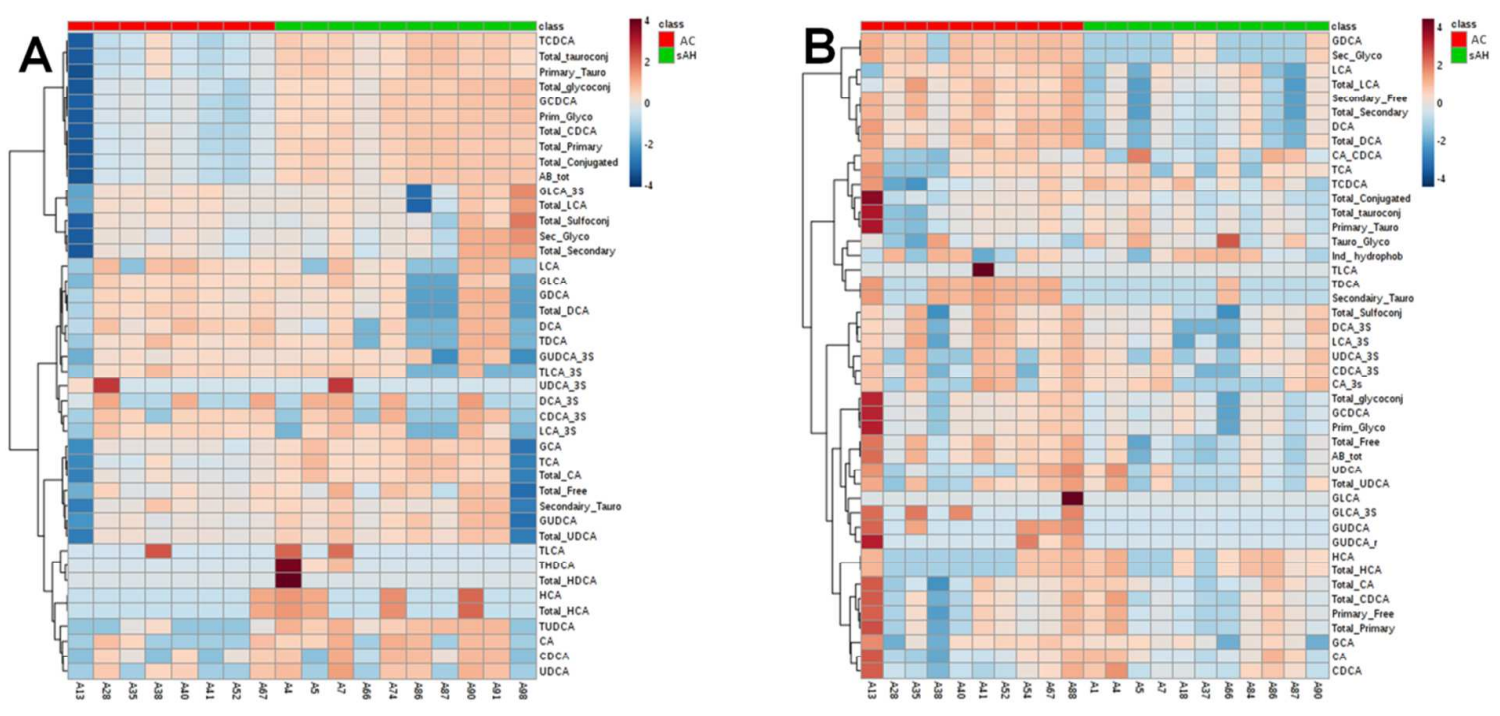

Supplemental Figure 1: Bile acids profiles in plasma (A) and feces (B). The relative amounts of bile acids are displayed as a heatmap (values are pareto and $\log 2$ scaled).
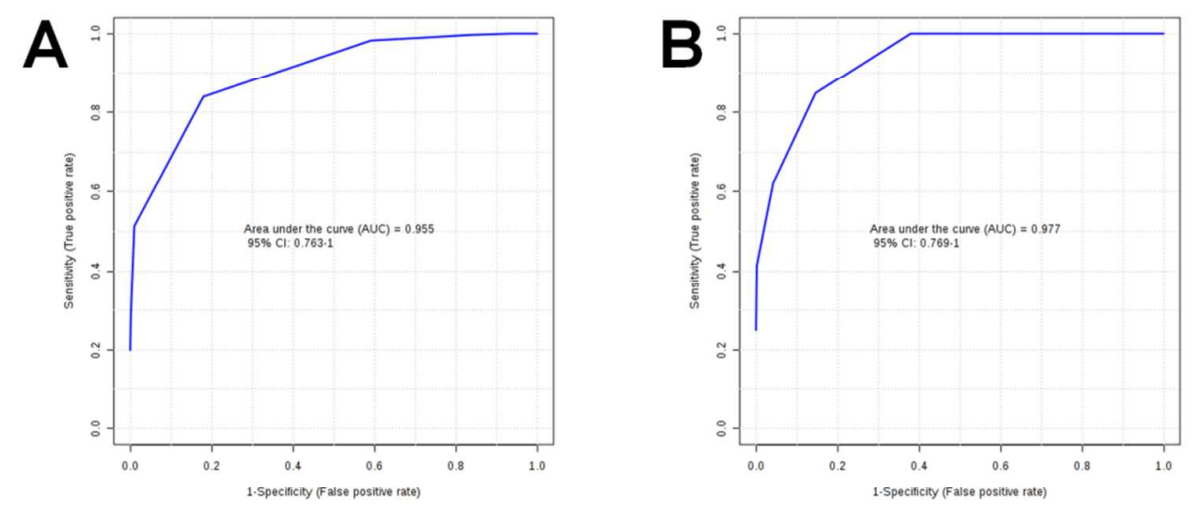

Supplemental figure 2: ROC curves showing that plasma $(\mathbf{A})$ and feces (B) bile acids are able to discriminate Cir_sAH patients from Cir_noAH patients (average AUROC $=0.955$ and 0.977 respectively) 